\title{
UNIQUENESS OF 1D GENERALIZED BI-SCHRÖDINGER FLOW
}

\author{
EIJI ONODERA
}

\begin{abstract}
We establish the uniqueness of a smooth generalized bi-Schrödinger flow from the one-dimensional flat torus into a compact locally Hermitian symmetric space. The governing equation, which is satisfied by sections of the pull-back bundle induced from the flow, is a fourth-order nonlinear dispersive partial differential equation with loss of derivatives. To show the uniqueness, we adopt an extrinsic approach to compare two solutions via an isometric embedding into an ambient Euclidean space. We introduce an energy modifying the classical $\mathrm{H}^{2}$-energy for the difference of two solutions, the detailed estimate of which enables us to eliminate the difficulty of the loss of derivatives. In particular, we demonstrate how to decide the form of the modification by exploiting the geometric structure of the locally Hermitian symmetric space.
\end{abstract}

\section{INTRODUCTION}

The so-called generalized bi-Schrödinger flow equation for maps from a Riemannian manifold into Kähler manifold was recently introduced by Ding and Wang in [7] in the following way: Let $(M, g)$ be an $m$-dimensional Riemannian manifold with a metric $g$ and let $(N, J, h)$ be a $2 n$-dimensional Kähler manifold with the complex structure $J$ and a Kähler metric $h$. Let $\alpha, \beta, \gamma \in \mathbb{R}$ be constants where $\beta \neq 0$. The energy functional $E_{\alpha, \beta, \gamma}(u)$ for smooth maps $u:(M, g) \rightarrow(N, J, h)$ is defined by

$$
E_{\alpha, \beta, \gamma}(u):=\alpha E(u)+\beta E_{2}(u)+\gamma E_{\star}(u) .
$$

Here, $E(u)=\frac{1}{2} \int_{M}|\nabla u|^{2} d v_{g}$ and $E_{2}(u)=\frac{1}{2} \int_{M}|\tau(u)|^{2} d v_{g}$ are energy functionals whose critical points are respectively known as harmonic maps and bi-harmonic maps, where $\nabla u$ is a section of the vector-bundle $T^{*} M \otimes u^{-1} T N, \tau(u)$ is the tension field of $u$, and $d v_{g}$ is the volume form of $(M, g)$. The energy functional $E_{\star}(u)$ is defined by

$$
E_{\star}(u):=\int_{M} h\left(R^{N}\left(\nabla u, J_{u} \nabla u\right) J_{u} \nabla u, \nabla u\right) d v_{g},
$$

where $R^{N}(\cdot, \cdot)$ is the Riemann curvature tensor on $(N, J, h)$. A time-dependent map $u=$ $u(t, x):(-T, T) \times M \rightarrow N$ is called a generalized bi-Schrödinger flow from $(M, g)$ to $(N, J, h)$ if $u$ satisfies the following Hamiltonian gradient flow equation

$$
u_{t}=J_{u} \nabla E_{\alpha, \beta, \gamma}(u)
$$

on $(-T, T) \times M$ for some $T>0$. We can see (1.1) as a fourth-order extension of the well-known Schrödinger map flow equation - a second-order geometric dispersive partial differential equation(PDE) - formulated by

$$
u_{t}=J_{u} \tau(u)
$$

2000 Mathematics Subject Classification. 53C44, 35G61, 53C21, 35Q35, 35Q40, 35Q55.

Key words and phrases. Generalized bi-Schrödinger flow; Locally Hermitian symmetric space; Dispersive partial differential equation; Uniqueness of a solution . 
which is just (1.1) under the assumption $(\alpha, \beta, \gamma)=(-1,0.0)$. For more details, see [7].

Another unified formulation of (1.1) was derived by the present author in [29] for timedependent maps from the real line $\mathbb{R}$ or the one-dimensional flat torus $\mathbb{T}:=\mathbb{R} / 2 \pi \mathbb{Z}$ into a locally Hermitian symmetric space. Let $(N, J, h)$ be a locally Hermitian symmetric space, which is a complex manifold characterized by the condition $\nabla^{N} J=\nabla^{N} R^{N}=0$. Here $\nabla^{N}$ denotes the Levi-Civita condition on $(N, J, h)$. Throughout this paper, we adopt the definition of $R^{N}$ by $R^{N}(X, Y) Z:=\nabla_{X}^{N} \nabla_{Y}^{N} Z-\nabla_{Y}^{N} \nabla_{X}^{N} Z-\nabla_{[X, Y]}^{N} Z$ for any $X, Y, Z \in \Gamma(T N)$. Then, the result of [29] shows (1.1) for time-dependent maps $u$ from $\mathbb{R}$ or $\mathbb{T}$ into $(N, J, h)$ can be written by

$$
\begin{aligned}
u_{t}= & \beta J_{u} \nabla_{x}^{3} u_{x}-\alpha J_{u} \nabla_{x} u_{x} \\
& +(\beta+8 \gamma) R^{N}\left(\nabla_{x} u_{x}, u_{x}\right) J_{u} u_{x}-12 \gamma R^{N}\left(J_{u} u_{x}, u_{x}\right) \nabla_{x} u_{x} .
\end{aligned}
$$

Here, $u_{t}=d u\left(\frac{\partial}{\partial t}\right), u_{x}=d u\left(\frac{\partial}{\partial x}\right), d u$ denotes the differential of $u, \nabla_{x}$ denotes the covariant derivative along $u$ with respect to $x, J_{u}$ denotes the complex structure at $u=u(t, x) \in N$.

Restricting ourselves to the case $(N, J, h)$ is the canonical 2 -sphere $\mathbb{S}^{2}$ with $\alpha=-1$, we find (1.3) arises in mathematical physics. Indeed, the $\mathbb{S}^{2}$-valued model (1.3) in this context is derived by a geometric reformulation of a continuum limit of the Heisenberg spin chain systems with nearest neighbor bilinear and bi-quadratic exchange interactions([18]). One can consult with [29, Section 2.2] for the reformulation of the physical model as (1.3). Moreover, the $\mathbb{S}^{2}$-valued model (1.3) also occurs in relation with the so-called Fukumoto-Moffatt model equation([11, 12]) describing the motion of a vortex filament in an incompressible prefect fluid in $\mathbb{R}^{3}$. In addition, by [11, 12,18$]$, the $\mathbb{S}^{2}$-valued model is known to be completely integrable under the additional assumption $\beta=-8 \gamma$, in that it has infinitely number of conservation laws.

In this paper, letting $(N, J, h)$ be a compact locally Hermitian symmetric space and restricting the spatial domain to $\mathbb{T}$, we consider the following initial value problem

$$
\begin{aligned}
& u_{t}=a J_{u} \nabla_{x}^{3} u_{x}+\lambda J_{u} \nabla_{x} u_{x}+b R^{N}\left(\nabla_{x} u_{x}, u_{x}\right) J_{u} u_{x}+c R^{N}\left(J_{u} u_{x}, u_{x}\right) \nabla_{x} u_{x}, \\
& u(0, x)=u_{0}(x),
\end{aligned}
$$

where a map $u=u(t, x): \mathbb{R} \times \mathbb{T} \rightarrow N$ is the solution being a flow of closed curve on $N$ and $u_{0}=u_{0}(x): \mathbb{T} \rightarrow N$ is the given initial closed curve on $N$. Moreover, $a \neq 0, b, c, \lambda$ are supposed to be real constants so that (1.4) is handled as a fourth-order nonlinear dispersive PDE. Obviously, the equation (1.4) with $(a, \lambda, b, c)=(\beta,-\alpha, \beta+8 \gamma,-12 \gamma)$ is nothing but (1.3). In other words, (1.4) slightly extends $(1.3)$ in the sense the relation $c=3(a-b) / 2$ is not imposed among the constants $a, b, c$.

1.1. Main results and related known results. The purpose of our research was to solve (1.4)-(1.5). This is a fundamental problem in the theory of PDEs. Moreover, making the relationship between the solvablity and the geometric setting of $(N, J, h)$ clear is fascinating also from the viewpoint of geometric analysis. In this part, we state the known results in this direction and our main results in this paper.

The local and global well-posedness for the Schrödinger map flow equation (1.2) with data in Sobolev spaces has been studied extensively by many authors. We do not attempt to survey all the results, but refer to [2, 3, 8, 9, 17, 20, 23, 30, 32, 37, 43] for more details. We note that the local existence and uniqueness results can be obtained under the Kähler condition $\nabla^{N} J=0$ on $(N, J, h)$ by exploiting the classical geometric energy method based on the integration by parts and the Sobolev embedding. If $\nabla^{N} J \neq 0$, then the so-called 
a loss of derivative occurs. In other words, the classical energy method breaks down due to the presence of a bad term coming from $\nabla^{N} J \neq 0$. Fortunately however, the difficulty was overcame in [3] by developing the energy method with a gauge transformation acting on sections of the pull-back bundle $u^{-1} T N$. In addition, some third-order generalizations of (1.2) have also been investigated in [5, 24, 25, 26, 35, 38, 39, 40]. We do not attempt to state the detail, but stress that the classical geometric energy method still works to obtain local existence and uniqueness results under the Kähler condition $\nabla^{N} J=0$.

In contrast, for our equation (1.4), we find the difficulty of loss of derivatives occurs even if $\nabla^{N} J=0$ holds. In general, the solvability of the initial value problem for a dispersive PDE essentially depends on the structure of the derivatives of the solution in the equation (see, e.g, [1, 4, 21, 22, 41]). Thus the crucial part of our problem is to reveal the relationship between the structure and the setting of $(N, J, h)$. The procedure becomes harder as the spatial order of the equation becomes higher, and the fourth-order case is the first one we encounter the difficulty of loss of derivatives under $\nabla^{N} J=0$. Furthermore, as the spatial domain $\mathbb{T}$ is compact, the so-called dispersive smoothing effect inherited to the solution on $\mathbb{R}$ - which was useful to compensate the loss of derivatives completely as in [6] - is absent in our setting. Therefore, a stronger geometric structure of $(N, J, h)$ is required even to establish local existence results.

Having the above in mind, we state the known results on $(1.4)$ in this direction. Guo, Zeng, and $\mathrm{Su}$ in [13] showed the local existence of weak solutions to (1.4)-(1.5) when $N=\mathbb{S}^{2}$ with $\lambda=1, a \neq 0, c=3(a-b) / 2$, and $b=0$. Note that the equation (1.4) in the setting without $b=0$ corresponds to the $\mathbb{S}^{2}$-valued physical model of continuum limit of the Heisenberg spin chain systems([18]) as stated above. The additional assumption $b=0$ corresponds to the completely integrable condition and the proof in [18] is essentially based on a conservation law which is absent unless $b=0$ is imposed. After that, the present author [27] showed the local existence and uniqueness of a smooth solution to (1.4)-(1.5) when $N=\mathbb{S}^{2}$ with $\lambda=1, a \neq 0$ and $c=3(a-b) / 2$ without any assumptions on the constants. In [28], he furthermore extended the results to the case $N$ is a compact Riemann surface with constant Gaussian curvature, without any additional assumptions on the constants except for $a \neq 0$.

Remark 1.1. In more detail, the equation handled in [28] stated above is formulated by

$$
u_{t}=b_{1} J_{u} \nabla_{x}^{3} u_{x}+b_{2} J_{u} \nabla_{x} u_{x}+b_{3} h\left(u_{x}, u_{x}\right) J_{u} \nabla_{x} u_{x}+b_{4} h\left(\nabla_{x} u_{x}, u_{x}\right) J_{u} u_{x}
$$

where $b_{1}(\neq 0), b_{2}, b_{3}, b_{4} \in \mathbb{R}$ are constants. This is different from (1.4) unless $(N, J, h)$ is a Riemann surface with constant Gaussian curvature. Moreover, as far as (1.6) is considered, it is unlikely that we can extend the local existence result to $(N, J, h)$ wider than the class of compact Riemann surfaces with constant curvature. This was first pointed out by Chihara in [4] from the theory of $L^{2}$-well-posedness for linear dispersive PDE systems, where the case of the Riemann surface as $(N, J, h)$ was discussed. The present author also investigated (1.6) in case of higher-dimensional manifolds, and found that local existence result still holds as far as $(N, J, h)$ is a compact $2 n$-dimensional Kähler manifold with constant non-vanishing sectional curvature (in the sense of a real manifold). However, it is meaningless, because the class of such manifolds is known to be the empty set without $n=1$ (see, e.g., [15, Theorem 7.1.]). Note again the strong obstruction is true only of (1.6), and not of (1.4).

Let us go back to our problem (1.4)-(1.5). Recently, the present author in [29] showed only the local existence of the solution to (1.4)-(1.5) for time-dependent maps into a compact locally Hermitian symmetric space. Indeed, by the mix of the parabolic regularization and 
the geometric energy method combined with a gauge transformation acting on $\Gamma\left(u^{-1} T N\right)$, he showed the following:

Theorem 1.2 ([29], Theorem 1.3.). Suppose that $(N, J, h)$ is a compact locally Hermitian symmetric space. Let $k$ be an integer satisfying $k \geqslant 4$. Then for any $u_{0} \in C(\mathbb{T} ; N)$ satisfying $u_{0 x} \in H^{k}(\mathbb{T} ; T N)$, there exists $T=T\left(\left\|u_{0 x}\right\|_{H^{4}(\mathbb{T} ; T N)}\right)>0$ such that (1.4)(1.5) has a solution $u \in C([-T, T] \times \mathbb{T} ; N)$ satisfying $u_{x} \in L^{\infty}\left(-T, T ; H^{k}(\mathbb{T} ; T N)\right) \cap$ $C\left([-T, T] ; H^{k-1}(\mathbb{T} ; T N)\right)$.

Here and hereafter, $\Gamma\left(u^{-1} T N\right)$ denotes the set of all sections of the pull-back bundle $u^{-1} T N$, and $H^{k}(\mathbb{T} ; T N)$ is defined to be the set of all sections $V \in \Gamma\left(u^{-1} T N\right)$ such that

$$
\|V\|_{H^{k}(\mathbb{T} ; T N)}:=\sum_{\ell=0}^{k} \int_{\mathbb{T}} h\left(\nabla_{x}^{\ell} V(x), \nabla_{x}^{\ell} V(x)\right) d x<\infty .
$$

It is natural to investigate whether the uniqueness of the solution to $(1.4)-(1.5)$ holds or not. The question is rather challenging, since how to apply the condition $\nabla^{N} R^{N}=0$ is unclear, unlikely to the proof of existence. Indeed, there are no other uniqueness results except for the very limited case of $(N, J, h)$ as stated above. The purpose of this paper is to establish how to apply $\nabla^{N} R^{N}=0$ and to prove the uniqueness. Our main result is now stated as follows:

Theorem 1.3. Suppose that $(N, J, h)$ is a compact locally Hermitian symmetric space. Let $k$ be an integer satisfying $k \geqslant 5$. Let $u$ and $v$ be solutions to $(1.4)-(1.5)$ in Theorem 1.2 Then it follows that $u=v$ on $[-T, T] \times \mathbb{T}$.

Remark 1.4. Let $u$ be a solution to (1.4)-(1.5) with regularity in Theorem 1.2 . Once the uniqueness is established, then we can recover the time-continuity of the highest derivative $\nabla_{x}^{k} u_{x}$ in $L^{2}$, by applying the weak time-continuity in $L^{2}$ and the energy estimate for $\left\|u_{x}(t)\right\|_{H^{k}(\mathbb{T} ; T N)}^{2}$ established in [29]. This shows $u_{x} \in C\left([-T, T] ; H^{k}(\mathbb{T} ; T N)\right)$. The argument is now standard, and hence we omit the detail.

Remark 1.5. Our proof of Theorem 1.3 works without modifications even if we replace $\mathbb{T}$ with $\mathbb{R}$. Nonetheless, no originality is claimed here, because the method previously established in [6] based on the dispersive smoothing effect works to prove in the case of $\mathbb{R}$.

Remark 1.6. The class of compact locally Hermitian symmetric spaces as $(N, J, h)$ includes all compact Kähler manifolds of constant holomorphic sectional curvature and compact Hermitian symmetric spaces, as well as compact Riemann surfaces with constant curvature. We should mention that the authors in [7] obtained the explicit formulation of (1.1) for timedependent maps from $\mathbb{R}$ or $\mathbb{T}$ into three types of Hermitian symmetric spaces as $(N, J, h)$ by using the Lie bracket in the symmetric Lie algebra of $N$. In particular, they obtained the explicit formulation when $N$ is a compact Kähler Grassmannian manifold $G_{n, k}$ for $n, k$ with $1 \leqslant k \leqslant n-1$. Though the formulation of the equation is seemingly different from (1.4), the uniqueness result for the equation also falls within the scope of Theorem 1.3 .

Remark 1.7. In [7], the equivalence of (1.1) for time-dependent maps from $\mathbb{R}$ into $N=G_{n, k}$ and a fourth-order nonlinear dispersive PDE for $k \times(n-k)$-complex-matrix-valued functions is also discussed. Particularly in the case $N=G_{2,1}$, corresponding equation for matrixvalued functions is just a (single) fourth-order semilinear dispersive PDE for complex-valued functions, and the well-posedness of the initial value problem in a Sobolev space was established by Segata in [33], including the case the spatial domain is $\mathbb{T}$. In the higher-dimensional 
case of $N$ except for $N=G_{2,1}$, we can see the corresponding equation for matrix-valued functions as a system of nonlinear dispersive PDEs including a nonlocal nonlinearity, which is more attractive in the interface of geometry and analysis of nonlinear dispersive PDEs. To the best of the author's knowledge, however, there are no results on the solvability of their initial value problem. If the equivalence holds also on the spatial domain $\mathbb{T}$, then Theorems 1.2 and 1.3 automatically give the local existence and uniqueness results on the dispersive PDEs for matrix-valued functions. Although it seems to be a nontrivial matter to show the equivalence, we are strongly convinced that the insights obtained in this paper give rise to valuable information about how to handle the dispersive PDEs for matrix-valued functions.

1.2. Key of the proof. In this part, we state the key idea of the proof of Theorem 1.3 after reviewing the proof of Theorem 1.2 briefly.

Theorem 1.2 on the local existence of a solution was proved by an intrinsic approach in [29]. To state the key observation, suppose $u$ is a smooth solution to (1.4)-(1.5). Then the equation satisfied by $\nabla_{x}^{k} u_{x}$ with $k \geqslant 4$ turns out to be described by

$$
\left(\nabla_{t}-a J_{u} \nabla_{x}^{4}-d_{1} P_{1} \nabla_{x}^{2}-d_{2} P_{2} \nabla_{x}\right) \nabla_{x}^{k} u_{x}=O\left(\sum_{m=0}^{k+2}\left|\nabla_{x}^{m} u_{x}\right|_{h}\right),
$$

where $|\cdot|_{h}=\{h(\cdot, \cdot)\}^{1 / 2}$, and $d_{1}$ and $d_{2}$ are real constants depending on $a, b, c, k$, and

$$
P_{1} Y:=R^{N}\left(Y, J_{u} u_{x}\right) u_{x} \quad \text { and } \quad P_{2} Y:=R^{N}\left(J_{u} \nabla_{x} u_{x}, u_{x}\right) Y
$$

respectively for any $Y \in \Gamma\left(u^{-1} T N\right)$. From (1.7), we find the classical energy estimate for $\left\|u_{x}\right\|_{H^{k}(\mathbb{T} ; T N)}^{2}$ breaks down because $d_{1} P_{1} \nabla_{x}^{2}$ and $d_{2} P_{2} \nabla_{x}$ cause loss of derivatives. Fortunately however, the difficulty was overcame in [29] by the geometric energy method combined with a gauge transformation acting on $\Gamma\left(u^{-1} T N\right)$. Observing the method in [29] in more detail, we find that the reason we can construct the gauge transformation comes from the following good properties:

(A) $\left(J_{u} P_{1}+P_{1} J_{u}\right) J_{u}$ is skew-symmetric on $\Gamma\left(u^{-1} T N\right)$.

(B) $P_{2}$ has a potential in the sense $P_{2}=\left(\nabla_{x} \widetilde{P}\right)$ where $\widetilde{P}=\frac{1}{2} R^{N}\left(J_{u} u_{x}, u_{x}\right)$.

We call them a "good structure" of (1.4) in this paper. We note also that the right hand side of (1.7) also includes $\nabla_{x}^{2}\left(\nabla_{x}^{k} u_{x}\right)$ and $\nabla_{x}\left(\nabla_{x}^{k} u_{x}\right)$, but loss of derivatives do not occur from the part thanks to the assumption $\nabla^{N} R^{N}=\nabla^{N} J=0$. It is unlikely that we can relax the assumption $\nabla^{N} R^{N}=0$, since the assumption seems to correspond to the constant curvature condition on the equation in [28]. Indeed, if we let $\nabla^{N} R^{N} \neq 0$, then (1.7) involves a skewsymmetric first-order derivative of the form $\left(\nabla_{x} R^{N}\right)\left(J_{u} u_{x}, u_{x}\right) \nabla_{x}\left(\nabla_{x}^{k} u_{x}\right)$, which is the worst term we cannot handle in the energy estimate.

Now we turn our attention to the proof of Theorem 1.3. To state the key of the proof simply, suppose $u$ and $v$ are sufficiently smooth solutions to (1.4)-(1.5) with same initial data. It suffices to show $u=v$. Since their difference is not defined on $N$ directly, we fix an isometric embedding $w$ from $(N, J, h)$ into some ambient Euclidean space $\mathbb{R}^{d}$ with sufficiently large integer $d$, and define

$$
Z:=U-V, \quad U:=w \circ u, \quad V:=w \circ v,
$$

as vector-valued functions with values in $\mathbb{R}^{d}$. However, then, the extrinsic formulation for the equation satisfied by $U$ becomes highly nonlinear (quasilinear) fourth-order dispersive equation involving $\partial_{x}^{3} U$ as well as $\partial_{x}^{2} U, \partial_{x} U$ in the nonlinearity and the good structure such as (1.7) is lost from the equation satisfied by $Z$ and the derivatives in $x$. Nonetheless, we 
expect that the good structure still remain at least in the tangential component. Having them in mind, we decompose the equation satisfied by the second derivative of $Z$ in $x$ into the tangential component in $d w\left(\Gamma\left(u^{-1} T N\right)\right)$ and normal component in $\left(d w\left(\Gamma\left(u^{-1} T N\right)\right)^{\perp}\right.$, and exploit the good structure hidden in $d w\left(\Gamma\left(u^{-1} T N\right)\right)$ to derive the estimate. The normal component is estimated by making use of some properties of the second fundamental form on $N$. More precisely, setting

$$
\mathcal{U}:=d w_{u}\left(\nabla_{x} u_{x}\right), \quad \mathcal{V}:=d w_{v}\left(\nabla_{x} v_{x}\right), \quad \mathcal{W}:=\mathcal{U}-\mathcal{V},
$$

we compute the equation satisfied by $Z, Z_{x}$ and $\mathcal{W}$ (not by $\partial_{x}^{2} Z$ in order to make the computation a little simpler). Particularly, the results of the computation for $\mathcal{W}$ is as follows:

$$
\mathcal{W}_{t}=a \partial_{x}^{2}\left\{J(U) \partial_{x}^{2} \mathcal{W}\right\}+\mathcal{N}\left(\partial_{x} \mathcal{W}, \partial_{x}^{2} \mathcal{W}, \partial_{x}^{3} \mathcal{W}\right)+\mathcal{O}\left(|Z|+\left|Z_{x}\right|+|\mathcal{W}|\right),
$$

where for each $(t, x), J(U(t, x)): \mathbb{R}^{d} \rightarrow \mathbb{R}^{d}$ is a map behaving as an almost complex structure on $d w_{u(t, x)}\left(T_{u(t, x)} N\right)$, and the nonlinearity $\mathcal{N}$ is an $\mathbb{R}^{d}$-valued function involving $\partial_{x}^{3} \mathcal{W}, \partial_{x}^{2} \mathcal{W}, \partial_{x} \mathcal{W}$. The explicit expression will be given by (3.43) (see also Proposition 3.1), and all the geometric notion used to describe (3.43) will be defined in Section 2. Looking at (3.43), 3.2), and the classical energy estimate (3.64) coming from them, we notice that the loss of derivatives in the classical energy estimate for $\mathcal{W}$ comes only from the linear combination of

$$
R\left(\partial_{x}^{2} \mathcal{W}, J(U) U_{x}\right) U_{x} \text { and } R\left(J(U) \mathcal{U}, U_{x}\right) \partial_{x} \mathcal{W}
$$

where the definition of $R$ will be given in $(2.13)$ in Section 2 , Then, motivated by the method in [4], we can choose a suitable gauge transformed function $\widetilde{W}$, which is formally written by

$$
\widetilde{W}=\mathcal{W}+\left(\Psi_{1}+\Psi_{2}\right) Z_{x x}
$$

where $\Psi_{1}$ and $\Psi_{2}$ will be defined in Remark 4.1 in Section 4, and they behave as pseudodifferential operators of order -2 . We can handle as $\widetilde{\mathcal{W}}=\mathcal{W}+\mathcal{O}(|Z|)$ in many situations. Moreover, we stress that the commutator of $\Psi_{1}$ (resp. $\Psi_{2}$ ) and the principal part $a \partial_{x}^{2}\left\{J(U) \partial_{x}^{2}\right\}$ effectively works to eliminate the part $R\left(\partial_{x}^{2} \mathcal{W}, J(U) U_{x}\right) U_{x}\left(\operatorname{resp} . R\left(J(U) \mathcal{U}, U_{x}\right) \partial_{x} \mathcal{W}\right)$. By using them, we can get the desired energy estimate for $\widetilde{D}(t)^{2}:=\|Z(t)\|_{L^{2}}^{2}+\left\|Z_{x}(t)\right\|_{L^{2}}^{2}+$ $\|\widetilde{\mathcal{W}}(t)\|_{L^{2}}^{2}$ so that we obtain $Z=0$, which implies $u=v$. Here, $\|\cdot\|_{L^{2}}$ denotes the standard $L^{2}$-norm for $\mathbb{R}^{d}$-valued functions on $\mathbb{T}$ (see Section 3.2). The assumption $k \geqslant 5$ comes from the requirement for the energy estimate to make sense, which slightly improves the previous one $k \geqslant 6$ imposed in [27, 28].

Remark 1.8. The extrinsic approach to uniqueness results via the isometric embedding into $\mathbb{R}^{d}$ has been adopted in broad range of geometric PDEs for maps into manifolds: Harmonic (or Biharmonic) map heat flow equation, Wave (or Biwave) map equation, Schrödinger map flow equation and the third- or fourth-order analogous dispersive curve flow equations, and so on. See, e.g., [5, 6, 8, 10, 14, 19, 29, 34, 40] and references therein. In particular, we mention again that the result in [29] shows Theorem 1.3 if we assume that $(N, J, h)$ is a compact Riemann surface with constant Gaussian curvature $S$. However, the argument of the proof breaks down without the assumption, since it is essentially based on the property

$$
R^{N}\left(Y_{1}, Y_{2}\right) Y_{3}=S\left(h\left(Y_{2}, Y_{3}\right) Y_{1}-h\left(Y_{2}, Y_{3}\right) Y_{1}\right) \quad\left(Y_{1}, Y_{2}, Y_{3} \in \Gamma\left(u^{-1} T N\right)\right) .
$$

Remark 1.9. The choice of $\widetilde{\mathcal{W}}$ is actually crucial in our proof as it is the part we exploit the good structure of (1.4). However, before doing this, we require obtaining Proposition 3.1. (3.43), and the estimate (3.64), which is more delicate issue, since we need to drop all the 
seemingly bad terms other than $R\left(\partial_{x}^{2} \mathcal{W}, J(U) U_{x}\right) U_{x}$ and $R\left(J(U) \mathcal{U}, U_{x}\right) \partial_{x} \mathcal{W}$ completely. To do this, we will develop the basic tools of computations on $\Gamma\left(u^{-1} T N\right)$ to those on the embedded submanifold in $\mathbb{R}^{d}$. More concretely, we will introduce the notion $R$ in Definition 2.5 and obtain some properties corresponding to those for $R^{N}$ (see Proposition 2.8). We point out that we can apply the assumption $\nabla^{N} R^{N}=0$ as (2.24). Moreover, we will obtain the connection between $R$ and the second fundamental form on $N$ in Proposition 2.7, which will be useful to compare the difference $d w_{u}\left(a \nabla_{x}^{3} u_{x}\right)-d w_{v}\left(a \nabla_{x}^{3} v_{x}\right)$ in Section 3 . Some other useful geometric properties applied in this paper also will be collected in Section 2.

The organization of the paper is as follows: In Section 2, we collect geometric notion and tools of computation used in this paper. In Section 3 divided by two subsections, we obtain the classical energy estimate for $\mathcal{W}$ by computing the equation satisfied by $\mathcal{W}$. In Section 4 based on the results in Section 3, we complete the proof of Theorem 1.3 .

\section{Geometric Preliminaries}

In this section, we collect some basic material of Riemannian geometry for maps into a locally Hermitian symmetric space, which will be used in Section 3 and 4 .

Let $w$ be an isometric embedding from $(N, J, h)$ into some Euclidean space $\mathbb{R}^{d}$ with sufficiently large integer $d$. For fixed $p \in N$, we consider the orthogonal decomposition $\mathbb{R}^{d}=d w_{p}\left(T_{p} N\right) \oplus\left(d w_{p}\left(T_{p} N\right)\right)^{\perp}$, where $d w_{p}: T_{p} N \rightarrow T_{w o p} \mathbb{R}^{d} \cong \mathbb{R}^{d}$ is the differential of $w$ at $p \in N$ and $\left(d w_{p}\left(T_{p} N\right)\right)^{\perp}$ is the orthogonal complement of $d w_{p}\left(T_{p} N\right)$ in $\mathbb{R}^{d}$. We denote the orthogonal projection mapping of $\mathbb{R}^{d}$ onto $d w_{p}\left(T_{p} N\right)$ by $P(w \circ p)$ and define $N(w \circ p):=I_{d}-P(w \circ p)$, where $I_{d}$ is the identity mapping on $\mathbb{R}^{d}$. Moreover, we define $J(w \circ p)$ as an action on $\mathbb{R}^{d}$ by first projecting onto $d w_{p}\left(T_{p} N\right)$ and then applying the complex structure at $p \in N$. More precisely, we define $J(w \circ p)$ by

$$
J(w \circ p):=d w_{p} \circ J_{p} \circ d w_{w \circ p}^{-1} \circ P(w \circ p): \mathbb{R}^{d} \rightarrow d w_{p}\left(T_{p} N\right) .
$$

We can extend $P(\cdot), N(\cdot)$, and $J(\cdot)$ to a smooth linear operator on $\mathbb{R}^{d}$ so that $P(q), N(q)$, and $J(q)$ make sense for all $q \in \mathbb{R}^{d}$ following the argument in e.g. [23, pp.17]. Though $J(q)$ is not skew-symmetric and the square is not the minus identity in general, similar properties still hold if $q \in w(N)$. Indeed, from the definition of $P(\omega \circ p)$ and $J(\omega \circ p)$, it follows that

$$
\begin{aligned}
\left(P(w \circ p) Y_{1}, Y_{2}\right) & =\left(Y_{1}, P(w \circ p) Y_{2}\right), \\
\left(J(w \circ p) Y_{1}, Y_{2}\right) & =-\left(Y_{1}, J(w \circ p) Y_{2}\right), \\
(J(w \circ p))^{2} Y_{3} & =-P(w \circ p) Y_{3},
\end{aligned}
$$

for any $p \in N$ and $Y_{1}, Y_{2}, Y_{3} \in \mathbb{R}^{d}$. Here and hereafter, we will denote the inner product and the norm in $\mathbb{R}^{d}$ by $(\cdot, \cdot)$ and $|\cdot|$ respectively.

Let $u=u(t, x):[-T, T] \times \mathbb{T} \rightarrow(N, J, h)$ be a sufficiently smooth map into a $2 n$ dimensional Kähler manifold $(N, J, h)$, and set $U:=w \circ u$. For each $(t, x) \in[-T, T] \times \mathbb{T}$, let $\left\{\nu_{2 n+1}, \ldots, \nu_{d}\right\}$ denote a smooth local orthonormal frame field for the normal bundle $(d w(T N))^{\perp}$ near $U(t, x) \in w(N)$. As is well-known, for $Y \in \Gamma\left(u^{-1} T N\right), d w_{u}\left(\nabla_{x} Y\right)$ (resp. $\left.d w_{u}\left(\nabla_{t} Y\right)\right)$ is nothing but the $d w_{u}\left(T_{u} N\right)$-component of $\partial_{x}\left\{d w_{u}(Y)\right\}\left(\operatorname{resp} . \partial_{t}\left\{d w_{u}(Y)\right\}\right)$, that is,

$$
d w_{u}\left(\nabla_{x} Y\right)=\partial_{x}\left\{d w_{u}(Y)\right\}-\sum_{k=2 n+1}^{d}\left(\partial_{x}\left\{d w_{u}(Y)\right\}, \nu_{k}(U)\right) \nu_{k}(U)
$$




$$
\begin{aligned}
& =\partial_{x}\left\{d w_{u}(Y)\right\}+\sum_{k=2 n+1}^{d}\left(d w_{u}(Y), \partial_{x}\left\{\nu_{k}(U)\right\}\right) \nu_{k}(U) \\
& =\partial_{x}\left\{d w_{u}(Y)\right\}+\sum_{k=2 n+1}^{d}\left(d w_{u}(Y), D_{k}(U) U_{x}\right) \nu_{k}(U) \\
& =\partial_{x}\left\{d w_{u}(Y)\right\}+A(U)\left(d w_{u}(Y), U_{x}\right), \\
d w_{u}\left(\nabla_{t} Y\right) & =\partial_{t}\left\{d w_{u}(Y)\right\}+A(U)\left(d w_{u}(Y), U_{t}\right) .
\end{aligned}
$$

where $D_{k}:=\operatorname{grad} \nu_{k}$ for $k=2 n+1, \ldots, d$, and $A(q)(\cdot, \cdot):=\sum_{k=2 n+1}^{d}\left(\cdot, D_{k}(q) \cdot\right) \nu_{k}(q)$ is the second fundamental form at $q \in w(N)$. As is well-known, $A(q)(\cdot, \cdot): T_{q} w(N) \times T_{q} w(N) \rightarrow$ $\mathbb{R}^{d}$ is a symmetric bilinear map, and each $D_{k}(q)$ is also symmetric, i.e., $\left(D_{k}(q) V_{1}, V_{2}\right)=$ $\left(V_{1}, D_{k}(q) V_{2}\right)$ holds for any $V_{1}, V_{2} \in T_{q} w(N)$. Furthermore, we can also see $A(q)(\cdot, \cdot)$ as a map on $\mathbb{R}^{d} \times \mathbb{R}^{d}$ by setting $A(q)\left(V_{1}, V_{2}\right):=A(q)\left(P(q) V_{1}, P(q) V_{2}\right)$ for any $V_{1}, V_{2} \in \mathbb{R}^{d}$. In addition, we note that $J(U) A(U)\left(Y_{1}, Y_{2}\right)=0$ holds for any $Y_{1}, Y_{2}:[-T, T] \times \mathbb{T} \rightarrow \mathbb{R}^{d}$. This comes from the property

$$
J(U) \nu_{k}(U)=0 \quad(k=2 n+1, \ldots, d),
$$

which is obvious by definition. In what follows, we will often use these properties and sometimes without any comments. Moreover, we will sometimes use the expression $\sum_{k}$ and $\sum_{k, \ell}$ instead of $\sum_{k=2 n+1}^{d}$ and $\sum_{k=2 n+1}^{d} \sum_{\ell=2 n+1}^{d}$ respectively. Any confusion will not occur.

We define the operator $\partial_{x}(J(U))$ by $\partial_{x}(J(U)) Y:=\partial_{x}\{J(U) Y\}-J(U) \partial_{x} Y$ for any $Y$ : $[-T, T] \times \mathbb{T} \rightarrow \mathbb{R}^{d}$. The following proposition comes from the Kähler condition $\nabla^{N} J=0$ on $(N, J, h)$.

Proposition 2.1. For any $Y:[-T, T] \times \mathbb{T} \rightarrow \mathbb{R}^{d}$, we have

$$
\begin{aligned}
\partial_{x}(J(U)) Y & =\sum_{k}\left(Y, J(U) D_{k}(U) U_{x}\right) \nu_{k}(U)-\sum_{k}\left(Y, \nu_{k}(U)\right) J(U) D_{k}(U) U_{x} \\
& =-A(U)\left(J(U) Y, U_{x}\right)-\sum_{k}\left(Y, \nu_{k}(U)\right) J(U) D_{k}(U) U_{x} .
\end{aligned}
$$

The proof is given in [29, Lemma 3.3], and thus we omit the detail.

We next define the operator $\partial_{x}^{m}(A(U))$ for $m=1,2, \ldots$ inductively by

$$
\begin{aligned}
\partial_{x}^{m}(A(U))\left(Y_{1}, Y_{2}\right):= & \partial_{x}\left\{\partial_{x}^{m-1}(A(U))\left(Y_{1}, Y_{2}\right)\right\}-\partial_{x}^{m-1}(A(U))\left(\partial_{x} Y_{1}, Y_{2}\right) \\
& -\partial_{x}^{m-1}(A(U))\left(Y_{1}, \partial_{x} Y_{2}\right)
\end{aligned}
$$

for any $Y_{1}, Y_{2}:[-T, T] \times \mathbb{T} \rightarrow \mathbb{R}^{d}$.

Proposition 2.2. For any $Y_{1}, Y_{2}:[-T, T] \times \mathbb{T} \rightarrow \mathbb{R}^{d}$, we have

$$
\begin{aligned}
\partial_{x}(A(U))\left(Y_{1}, Y_{2}\right)= & \sum_{k}\left(P(U) Y_{1}, \partial_{x}\left(D_{k}(U) P(U)\right) Y_{2}\right) \nu_{k}(U) \\
& +\sum_{k}\left(\partial_{x}(P(U)) Y_{1}, D_{k}(U) P(U) Y_{2}\right) \nu_{k}(U)
\end{aligned}
$$




$$
\begin{gathered}
+\sum_{k}\left(P(U) Y_{1}, D_{k}(U) P(U) Y_{2}\right) D_{k}(U) U_{x} \\
J(U) \partial_{x}(A(U))\left(Y_{1}, Y_{2}\right)=\sum_{k}\left(P(U) Y_{1}, D_{k}(U) P(U) Y_{2}\right) J(U) D_{k}(U) U_{x} .
\end{gathered}
$$

Proof of Proposition 2.2. By a simple computation based on the definition of $\partial_{x}^{m}(A(U))$ and the property (2.7), we can easily check (2.9) and (2.10) hold.

Furthermore, by the same computation as we obtain Proposition 2.2 and by $(2.8)$, we get the following:

Proposition 2.3. For any $Y_{1}, Y_{2}:[-T, T] \times \mathbb{T} \rightarrow \mathbb{R}^{d}$, it follows that

$$
\begin{aligned}
\partial_{x} & (J(U)) \partial_{x}(A(U))\left(Y_{1}, Y_{2}\right) \\
= & -\sum_{k}\left(P(U) Y_{1}, \partial_{x}\left(D_{k}(U) P(U)\right) Y_{2}\right) J(U) D_{k}(U) U_{x} \\
& -\sum_{k}\left(\partial_{x}(P(U)) Y_{1}, D_{k}(U) P(U) Y_{2}\right) J(U) D_{k}(U) U_{x} \\
& -\sum_{k, \ell}\left(P(U) Y_{1}, D_{k}(U) P(U) Y_{2}\right)\left(D_{k}(U) U_{x}, \nu_{\ell}(U)\right) J(U) D_{\ell}(U) U_{\ell} \\
& -\sum_{k} A(U)\left(\left(P(U) Y_{1}, D_{k}(U) P(U) Y_{2}\right) J(U) D_{k}(U) U_{x}, U_{x}\right), \\
J( & U) \partial_{x}^{2}(A(U))\left(Y_{1}, Y_{2}\right) \\
= & 2 \sum_{k}\left(P(U) Y_{1}, \partial_{x}\left(D_{k}(U) P(U)\right) Y_{2}\right) J(U) D_{k}(U) U_{x} \\
& +2 \sum_{k}\left(\partial_{x}(P(U)) Y_{1}, D_{k}(U) P(U) Y_{2}\right) J(U) D_{k}(U) U_{x} \\
& +\sum_{k}\left(P(U) Y_{1}, D_{k}(U) P(U) Y_{2}\right) J(U) \partial_{x}\left\{D_{k}(U) U_{x}\right\} .
\end{aligned}
$$

The following properties on the Riemann curvature tensor $R^{N}$ on $(N, J, h)$ are well-known in Riemannian geometry:

Proposition 2.4. For any $Y_{1}, \ldots, Y_{4} \in \Gamma\left(u^{-1} T N\right)$, the following properties hold.

(i) $R^{N}\left(Y_{1}, Y_{2}\right)=-R^{N}\left(Y_{2}, Y_{1}\right)$,

(ii) $h\left(R^{N}\left(Y_{1}, Y_{2}\right) Y_{3}, Y_{4}\right)=h\left(R^{N}\left(Y_{3}, Y_{4}\right) Y_{1}, Y_{2}\right)=h\left(R^{N}\left(Y_{4}, Y_{3}\right) Y_{2}, Y_{1}\right)$,

(iii) $R^{N}\left(Y_{1}, Y_{2}\right) Y_{3}+R^{N}\left(Y_{2}, Y_{3}\right) Y_{1}+R^{N}\left(Y_{3}, Y_{1}\right) Y_{2}=0$,

(iv) $R^{N}\left(Y_{1}, Y_{2}\right) J_{u} Y_{3}=J_{u} R^{N}\left(Y_{1}, Y_{2}\right) Y_{3}$,

(v) $R^{N}\left(J_{u} Y_{1}, J_{u} Y_{2}\right) Y_{3}=R^{N}\left(Y_{1}, Y_{2}\right) Y_{3}$,

(vi) $R^{N}\left(J_{u} Y_{1}, Y_{2}\right) Y_{3}=-R^{N}\left(Y_{1}, J_{u} Y_{2}\right) Y_{3}=R^{N}\left(J_{u} Y_{2}, Y_{1}\right) Y_{3}$,

(vii) $\nabla_{x}\left\{R^{N}\left(Y_{1}, Y_{2}\right) Y_{3}\right\}=R^{N}\left(\nabla_{x} Y_{1}, Y_{2}\right) Y_{3}+R^{N}\left(Y_{1}, \nabla_{x} Y_{2}\right) Y_{3}+R^{N}\left(Y_{1}, Y_{2}\right) \nabla_{x} Y_{3}$.

Note that the property (vii) comes from $\nabla^{N} R^{N}=0$ imposed on $(N, J, h)$. These properties were effectively applied to show the local existence of the solution to (1.4)-(1.5) in [29]. On the other hand, in order to show the uniqueness, we will require using these properties after acting $d w_{u}$ on them. For this purpose, we now introduce the following: 
Definition 2.5. We define the operator $R$ by

$$
R\left(Y_{1}, Y_{2}\right) Y_{3}:=d w_{u}\left(R^{N}\left(d w_{U}^{-1}\left(P(U) Y_{1}\right), d w_{U}^{-1}\left(P(U) Y_{2}\right)\right) d w_{U}^{-1}\left(P(U) Y_{3}\right)\right)
$$

for any $Y_{1}, Y_{2}, Y_{3}:[-T, T] \times \mathbb{T} \rightarrow \mathbb{R}^{d}$.

By the definition, it is immediate to see

$$
\begin{aligned}
& R(P(U) \cdot, \cdot)=R(\cdot, P(U) \cdot)=R(\cdot, \cdot) P(U)=R(\cdot, \cdot), \\
& R(A(U)(\cdot, \cdot), \cdot)=R(\cdot, A(U)(\cdot, \cdot))=R(\cdot, \cdot) A(U)(\cdot, \cdot)=0 .
\end{aligned}
$$

On the local expression of $R$, let us recall the following:

Proposition 2.6. For any $\Xi_{1}, \Xi_{2}, \Xi_{3} \in \Gamma\left(u^{-1} T N\right)$, the following relation holds.

$$
\begin{aligned}
d w_{u}\left(R^{N}\left(\Xi_{1}, \Xi_{2}\right) \Xi_{3}\right)= & \sum_{k=2 n+1}^{d}\left(d w_{u}\left(\Xi_{3}\right), D_{k}(U) d w_{u}\left(\Xi_{2}\right)\right) P(U) D_{k}(U) d w_{u}\left(\Xi_{1}\right) \\
& -\sum_{k=2 n+1}^{d}\left(d w_{u}\left(\Xi_{3}\right), D_{k}(U) d w_{u}\left(\Xi_{1}\right)\right) P(U) D_{k}(U) d w_{u}\left(\Xi_{2}\right) .
\end{aligned}
$$

Proposition 2.6 is a kind of expression of the Gauss-Codazzi formula in Riemannian geometry, and the proof can be seen in [28, Lemma 3.5]. Using Proposition 2.6 with $\Xi_{i}=$ $d w_{U}^{-1}\left(P(U) Y_{i}\right) \in \Gamma\left(u^{-1} T N\right), i=1,2,3$, we have the following

Proposition 2.7. For any $Y_{1}, Y_{2}, Y_{3}:[-T, T] \times \mathbb{T} \rightarrow \mathbb{R}^{d}$, it follows that

$$
\begin{aligned}
R\left(Y_{1}, Y_{2}\right) Y_{3}:= & \sum_{k=2 n+1}^{d}\left(P(U) Y_{3}, D_{k}(U) P(U) Y_{2}\right) P(U) D_{k}(U) P(U) Y_{1} \\
& -\sum_{k=2 n+1}^{d}\left(P(U) Y_{3}, D_{k}(U) P(U) Y_{1}\right) P(U) D_{k}(U) P(U) Y_{2} .
\end{aligned}
$$

If $\Xi_{1}, \Xi_{2}, \Xi_{3} \in \Gamma\left(u^{-1} T N\right)$, then 2.13 with $P(U) Y_{i}=Y_{i}=d w_{u}\left(\Xi_{i}\right), i=1,2,3$, implies

$$
d w_{u}\left(R^{N}\left(\Xi_{1}, \Xi_{2}\right) \Xi_{3}\right)=R\left(d w_{u}\left(\Xi_{1}\right), d w_{u}\left(\Xi_{2}\right)\right) d w_{u}\left(\Xi_{3}\right) .
$$

Using them, we have the following properties for $R$ corresponding to (i) -(vii) in Proposition 2.4 for $R^{N}$.

Proposition 2.8. For any $Y_{1}, Y_{2}, Y_{3}, Y_{4}:[-T, T] \times \mathbb{T} \rightarrow \mathbb{R}^{d}$, the following properties hold.

$$
\begin{aligned}
& R\left(Y_{1}, Y_{2}\right)=-R\left(Y_{2}, Y_{1}\right) \\
& \left(R\left(Y_{1}, Y_{2}\right) Y_{3}, Y_{4}\right)=\left(R\left(Y_{3}, Y_{4}\right) Y_{1}, Y_{2}\right)=\left(R\left(Y_{4}, Y_{3}\right) Y_{2}, Y_{1}\right), \\
& R\left(Y_{1}, Y_{2}\right) Y_{3}+R\left(Y_{2}, Y_{3}\right) Y_{1}+R\left(Y_{3}, Y_{1}\right) Y_{2}=0, \\
& R\left(Y_{1}, Y_{2}\right) J(U) Y_{3}=J(U) R\left(Y_{1}, Y_{2}\right) Y_{3}, \\
& R\left(J(U) Y_{1}, J(U) Y_{2}\right) Y_{3}=R\left(Y_{1}, Y_{2}\right) Y_{3}, \\
& R\left(J(U) Y_{1}, Y_{2}\right) Y_{3}=-R\left(Y_{1}, J(U) Y_{2}\right) Y_{3}=R\left(J(U) Y_{2}, Y_{1}\right) Y_{3}, \\
& \partial_{x}\left\{R\left(Y_{1}, Y_{2}\right) Y_{3}\right\} \\
& =R\left(\partial_{x}\left\{P(U) Y_{1}\right\}, Y_{2}\right) Y_{3}+R\left(Y_{1}, \partial_{x}\left\{P(U) Y_{2}\right\}\right) Y_{3} \\
& \quad+R\left(Y_{1}, Y_{2}\right) \partial_{x}\left\{P(U) Y_{3}\right\}-A(U)\left(R\left(Y_{1}, Y_{2}\right) Y_{3}, U_{x}\right) .
\end{aligned}
$$


Proof of Proposition 2.8 It is easy to see (2.18) follows from the definition of $R$. It is obvious that (2.19), ..., (2.24) are obtained by acting $d w_{u}$ to (ii), .., (vii) in Proposition 2.4 respectively. We omit the detail, but state only how to obtain (2.24), as it corresponds to the key property (vii) coming from $\nabla^{N} R^{N}=0$. To obtain (2.24), note first that there exists $\Xi_{i} \in \Gamma\left(u^{-1} T N\right)$ such that $d w_{u}\left(\Xi_{i}\right)=P(U) Y_{i}$ for each $i=1,2,3$. From (vii) in Proposition 2.4, it follows that

$$
\nabla_{x}\left\{R^{N}\left(\Xi_{1}, \Xi_{2}\right) \Xi_{3}\right\}=R^{N}\left(\nabla_{x} \Xi_{1}, \Xi_{2}\right) \Xi_{3}+R^{N}\left(\Xi_{1}, \nabla_{x} \Xi_{2}\right) \Xi_{3}+R^{N}\left(\Xi_{1}, \Xi_{2}\right) \nabla_{x} \Xi_{3} .
$$

Using (2.5) with $Y=R^{N}\left(\Xi_{1}, \Xi_{2}\right) \Xi_{3}$, 2.17), and (2.14), we have

$$
\begin{aligned}
& d w_{u}\left(\nabla_{x}\left\{R^{N}\left(\Xi_{1}, \Xi_{2}\right) \Xi_{3}\right\}\right) \\
& =\partial_{x}\left\{d w_{u}\left(R^{N}\left(\Xi_{1}, \Xi_{2}\right) \Xi_{3}\right)\right\}+A(U)\left(d w_{u}\left(R^{N}\left(\Xi_{1}, \Xi_{2}\right) \Xi_{3}\right), U_{x}\right) \\
& =\partial_{x}\left\{R\left(d w_{u}\left(\Xi_{1}\right), d w_{u}\left(\Xi_{2}\right)\right) d w_{u}\left(\Xi_{3}\right)\right\}+A(U)\left(R\left(d w_{u}\left(\Xi_{1}\right), d w_{u}\left(\Xi_{2}\right)\right) d w_{u}\left(\Xi_{3}\right), U_{x}\right) \\
& =\partial_{x}\left\{R\left(P(U) Y_{1}, P(U) Y_{2}\right) P(U) Y_{3}\right\}+A(U)\left(R\left(P(U) Y_{1}, P(U) Y_{2}\right) P(U) Y_{3}, U_{x}\right) \\
& =\partial_{x}\left\{R\left(Y_{1}, Y_{2}\right) Y_{3}\right\}+A(U)\left(R\left(Y_{1}, Y_{2}\right) Y_{3}, U_{x}\right) .
\end{aligned}
$$

In the same way, we have

$$
\begin{aligned}
d w_{u} & \left(R^{N}\left(\nabla_{x} \Xi_{1}, \Xi_{2}\right) \Xi_{3}+R^{N}\left(\Xi_{1}, \nabla_{x} \Xi_{2}\right) \Xi_{3}+R^{N}\left(\Xi_{1}, \Xi_{2}\right) \nabla_{x} \Xi_{3}\right) \\
= & R\left(d w_{u}\left(\nabla_{x} \Xi_{1}\right), d w_{u}\left(\Xi_{2}\right)\right) d w_{u}\left(\Xi_{3}\right)+R\left(d w_{u}\left(\Xi_{1}\right), d w_{u}\left(\nabla_{x} \Xi_{2}\right)\right) d w_{u}\left(\Xi_{3}\right) \\
& +R\left(d w_{u}\left(\Xi_{1}\right), d w_{u}\left(\Xi_{2}\right)\right) d w_{u}\left(\nabla_{x} \Xi_{3}\right) \\
= & R\left(\partial_{x}\left\{d w_{u}\left(\Xi_{1}\right)\right\}+A(U)\left(d w_{u}\left(\Xi_{1}\right), U_{x}\right), d w_{u}\left(\Xi_{2}\right)\right) d w_{u}\left(\Xi_{3}\right) \\
& +R\left(d w_{u}\left(\Xi_{1}\right), \partial_{x}\left\{d w_{u}\left(\Xi_{2}\right)\right\}+A(U)\left(d w_{u}\left(\Xi_{2}\right), U_{x}\right)\right) d w_{u}\left(\Xi_{3}\right) \\
& +R\left(d w_{u}\left(\Xi_{1}\right), d w_{u}\left(\Xi_{2}\right)\right)\left(\partial_{x}\left\{d w_{u}\left(\Xi_{3}\right)\right\}+A(U)\left(d w_{u}\left(\Xi_{3}\right), U_{x}\right)\right) \\
= & R\left(\partial_{x}\left\{P(U) Y_{1}\right\}+A(U)\left(P(U) Y_{1}, U_{x}\right), P(U) Y_{2}\right) P(U) Y_{3} \\
& +R\left(P(U) Y_{1}, \partial_{x}\left\{P(U) Y_{2}\right\}+A(U)\left(P(U) Y_{2}, U_{x}\right)\right) P(U) Y_{3} \\
& +R\left(P(U) Y_{1}, P(U) Y_{2}\right)\left(\partial_{x}\left\{P(U) Y_{3}\right\}+A(U)\left(P(U) Y_{3}, U_{x}\right)\right) \\
= & R\left(\partial_{x}\left\{P(U) Y_{1}\right\}, Y_{2}\right) Y_{3}+R\left(Y_{1}, \partial_{x}\left\{P(U) Y_{2}\right\}\right) Y_{3}+R\left(Y_{1}, Y_{2}\right) \partial_{x}\left\{P(U) Y_{3}\right\},
\end{aligned}
$$

where in the final equality we used (2.15). Comparing them, we obtain (2.24).

We next set $\mathcal{U}=d w_{u}\left(\nabla_{x} u_{x}\right)$ as given in Introduction.

Definition 2.9. We define operators $B_{i}(U), i=1,2,3$, by

$$
\begin{aligned}
& B_{1}(U) Y:=R(Y, \mathcal{U}) J(U) U_{x}-R\left(Y, U_{x}\right) J(U) \mathcal{U}, \\
& B_{2}(U) Y:=R(Y, \mathcal{U}) J(U) U_{x}+R\left(Y, J(U) U_{x}\right) \mathcal{U}, \\
& B_{3}(U) Y:=R\left(Y, U_{x}\right) J(U) \mathcal{U}+R(Y, J(U) \mathcal{U}) U_{x}
\end{aligned}
$$

for any $Y:[-T, T] \times \mathbb{T} \rightarrow \mathbb{R}^{d}$.

Proposition 2.10. Each of $B_{i}(U)(i=1,2,3)$ is symmetric, that is,

$$
\left(B_{i}(U) Y, Z\right)=\left(Y, B_{i}(U) Z\right)
$$

holds for any $Y, Z:[-T, T] \times \mathbb{T} \rightarrow \mathbb{R}^{d}$. 
Proof of Proposition 2.10. For $i=1$, it follows that

$$
\begin{aligned}
\left(B_{1}(U) Y, Z\right)= & \left(R(Y, \mathcal{U}) J(U) U_{x}, Z\right)-\left(R\left(Y, U_{x}\right) J(U) \mathcal{U}, Z\right) \\
= & -\left(R\left(\mathcal{U}, J(U) U_{x}\right) Y, Z\right)-\left(R\left(J(U) U_{x}, Y\right) \mathcal{U}, Z\right) \\
& +\left(R\left(U_{x}, J(U) \mathcal{U}\right) Y, Z\right)+\left(R(J(U) \mathcal{U}, Y) U_{x}, Z\right) \quad(\because(2.20)) \\
= & \left.-\left(R\left(J(U) U_{x}, Y\right) \mathcal{U}, Z\right)+\left(R(J(U) \mathcal{U}, Y) U_{x}, Z\right) \quad(\because(2.18),(2.23))\right) \\
= & \left(R(Z, \mathcal{U}) J(U) U_{x}, Y\right)-\left(R\left(Z, U_{x}\right) J(U) \mathcal{U}, Y\right) \quad(\because(2.18),(2.19)) \\
= & \left(B_{1}(U) Z, Y\right) .
\end{aligned}
$$

For $i=2$ and $i=3$, 2.25) follows from (2.19).

Proposition 2.11. For any $Y:[-T, T] \times \mathbb{T} \rightarrow \mathbb{R}^{d}$, it follows that

$$
\begin{aligned}
& R(Y, \mathcal{U}) J(U) U_{x}=\frac{1}{2} R\left(J(U) \mathcal{U}, U_{x}\right) Y+\left(\frac{1}{2} B_{1}(U)+\frac{1}{4} B_{2}(U)+\frac{1}{4} B_{3}(U)\right) Y, \\
& R\left(Y, U_{x}\right) J(U) \mathcal{U}=\frac{1}{2} R\left(J(U) \mathcal{U}, U_{x}\right) Y-\left(\frac{1}{2} B_{1}(U)-\frac{1}{4} B_{2}(U)-\frac{1}{4} B_{3}(U)\right) Y .
\end{aligned}
$$

Proof of Proposition 2.11. Using $B_{i}(U)(i=1,2,3)$, we can rewrite as follows:

$$
\begin{array}{rl}
4 & R(Y, \mathcal{U}) J(U) U_{x} \\
= & 2 B_{1}(U) Y+2\left\{R(Y, \mathcal{U}) J(U) U_{x}+R\left(Y, U_{x}\right) J(U) \mathcal{U}\right\} \\
= & 2 B_{1}(U) Y+B_{2}(U) Y+B_{3}(U) Y+R(Y, \mathcal{U}) J(U) U_{x}-R\left(Y, J(U) U_{x}\right) \mathcal{U} \\
& +R\left(Y, U_{x}\right) J(U) \mathcal{U}-R(Y, J(U) \mathcal{U}) U_{x} .
\end{array}
$$

Here, it follows that

$$
\begin{aligned}
R(Y, \mathcal{U}) J(U) U_{x}+R\left(Y, U_{x}\right) J(U) \mathcal{U} \\
=-R\left(\mathcal{U}, J(U) U_{x}\right) Y-R\left(J(U) U_{x}, Y\right) \mathcal{U} \\
\left.\quad-R\left(U_{x}, J(U) \mathcal{U}\right) Y-R(J(U) \mathcal{U}, Y) U_{x} \quad(\because 2.20)\right) \\
\left.=2 R\left(J(U) \mathcal{U}, U_{x}\right) Y+R\left(Y, J(U) U_{x}\right) \mathcal{U}+R(Y, J(U) \mathcal{U}) U_{x} \quad(\because 2.18,2.23)\right)
\end{aligned}
$$

Combining (2.28) and 2.29), we have 2.26). In the same way, since

$$
4 R\left(Y, U_{x}\right) J(U) \mathcal{U}=-2 B_{1}(U) Y+2\left\{R(Y, \mathcal{U}) J(U) U_{x}+R\left(Y, U_{x}\right) J(U) \mathcal{U}\right\},
$$

we have (2.27).

Next, we set

$$
T(U):=J(U) \partial_{x}(A(U))\left(\cdot, U_{x}\right)=\sum_{k}\left(P(U) \cdot D_{k}(U) U_{x}\right) J(U) D_{k}(U) U_{x},
$$

the exact form of which has been obtained by (2.10) in Proposition 2.3. We here observe the expression of $\partial_{x}(T(U))$ defined by $\partial_{x}(T(U)) Y:=\partial_{x}\{T(U) Y\}-T(U) \partial_{x} Y$. By the definition and the expression (2.10), it is easy to find

$$
\begin{aligned}
\partial_{x}(T(U)) Y= & \sum_{k}\left(P(U) Y, \partial_{x}\left\{D_{k}(U) U_{x}\right\}\right) J(U) D_{k}(U) U_{x} \\
& +\sum_{k}\left(P(U) Y, D_{k}(U) U_{x}\right) \partial_{x}\left\{J(U) D_{k}(U) U_{x}\right\}
\end{aligned}
$$




$$
+\sum_{k}\left(\partial_{x}(P(U)) Y, D_{k}(U) U_{x}\right) J(U) D_{k}(U) U_{x}
$$

for any $Y:[-T, T] \times \mathbb{T} \rightarrow \mathbb{R}^{d}$. In fact, as an application of the tools stated above, we can obtain the following another expression.

Proposition 2.12. For any $Y:[-T, T] \times \mathbb{T} \rightarrow \mathbb{R}^{d}$, it follows that

$$
\begin{aligned}
\partial_{x}(T(U)) Y= & -R\left(J(U) \mathcal{U}, U_{x}\right) Y-\frac{1}{2}\left(B_{2}(U)+B_{3}(U)\right) Y \\
& -J(U) R\left(U_{x}, \partial_{x}(N(U)) Y\right) U_{x}-A(U)\left(J(U) R\left(U_{x}, Y\right) U_{x}, U_{x}\right) \\
& +\sum_{k} \partial_{x}\left(\left(U_{x}, D_{k}(U) U_{x}\right) J(U) D_{k}(U) P(U)\right) Y .
\end{aligned}
$$

Proof of Proposition 2.12 Using $J(U)=J(U) P(U)$, the symmetry of $D_{k}(U)$, and (2.16) with $Y_{1}=Y_{3}=U_{x}=P(U) U_{x}$ and $Y_{2}=Y$, we can deduce

$$
\begin{aligned}
T(U) Y & =J(U) \sum_{k}\left(P(U) Y, D_{k}(U) U_{x}\right) P(U) D_{k}(U) U_{x} \\
& =J(U) \sum_{k}\left(U_{x}, D_{k}(U) P(U) Y\right) P(U) D_{k}(U) U_{x} \\
& =J(U)\left\{R\left(U_{x}, Y\right) U_{x}+\sum_{k}\left(U_{x}, D_{k}(U) U_{x}\right) P(U) D_{k}(U) P(U) Y\right\} \\
& =J(U) R\left(U_{x}, Y\right) U_{x}+\sum_{k}\left(U_{x}, D_{k}(U) U_{x}\right) J(U) D_{k}(U) P(U) Y .
\end{aligned}
$$

By computing $\partial_{x}(T(U)) Y=\partial_{x}\{T(U) Y\}-T(U) \partial_{x} Y$ under the expression, we can obtain (2.32). We demonstrate only the computation related to the first term of the right hand side of (2.33) here. To begin with, it follows that

$$
\begin{aligned}
& \partial_{x}\left\{J(U) R\left(U_{x}, Y\right) U_{x}\right\}-J(U) R\left(U_{x}, \partial_{x} Y\right) U_{x} \\
& =\partial_{x}(J(U)) R\left(U_{x}, Y\right) U_{x}+J(U) \partial_{x}\left\{R\left(U_{x}, Y\right) U_{x}\right\}-J(U) R\left(U_{x}, \partial_{x} Y\right) U_{x} .
\end{aligned}
$$

Using (2.8) with $Y=R\left(U_{x}, Y\right) U_{x}$, and noting $R\left(U_{x}, Y\right) U_{x} \perp \nu_{k}(U)$, we see

$$
\partial_{x}(J(U)) R\left(U_{x}, Y\right) U_{x}=-A(U)\left(J(U) R\left(U_{x}, Y\right) U_{x}, U_{x}\right) .
$$

Using (2.24) with $Y_{1}=Y_{3}=U_{x}=P(U) U_{x}$ and $Y_{2}=Y$, we deduce

$$
\begin{aligned}
(\sharp):= & J(U) \partial_{x}\left\{R\left(U_{x}, Y\right) U_{x}\right\}-J(U) R\left(U_{x}, \partial_{x} Y\right) U_{x} \\
= & J(U) R\left(U_{x x}, Y\right) U_{x}+J(U) R\left(U_{x}, \partial_{x}\{P(U) Y\}\right) U_{x}+J(U) R\left(U_{x}, Y\right) U_{x x} \\
& -J(U) A(U)\left(R\left(U_{x}, Y\right) U_{x}, U_{x}\right)-J(U) R\left(U_{x}, \partial_{x} Y\right) U_{x} \\
= & J(U) R\left(U_{x x}, Y\right) U_{x}+J(U) R\left(U_{x}, Y\right) U_{x x}+J(U) R\left(U_{x}, \partial_{x}(P(U)) Y\right) U_{x} \\
& -J(U) R\left(U_{x}, N(U) \partial_{x} Y\right) U_{x}-J(U) A(U)\left(R\left(U_{x}, Y\right) U_{x}, U_{x}\right),
\end{aligned}
$$

where in the final equality we use $P(U)+N(U)=I_{d}$. By (2.5), we see $\mathcal{U}=U_{x x}+$ $A(U)\left(U_{x}, U_{x}\right)$. By (2.7), we see $J(U) A(U)(\cdot, \cdot)=0$. By (2.14) and $P(U) N(U)=0$, $R(\cdot, N(U) \cdot)=0$. By $P(U)+N(U)=I_{d}$, we see $\partial_{x}(P(U))=-\partial_{x}(N(U))$. Using them, and using 2.18) and 2.21, we deduce

$$
(\sharp)=J(U) R(\mathcal{U}, Y) U_{x}+J(U) R\left(U_{x}, Y\right) \mathcal{U}-J(U) R\left(U_{x}, \partial_{x}(N(U)) Y\right) U_{x}
$$




$$
=-R(Y, \mathcal{U}) J(U) U_{x}-R\left(Y, U_{x}\right) J(U) \mathcal{U}-J(U) R\left(U_{x}, \partial_{x}(N(U)) Y\right) U_{x} .
$$

Furthermore, applying 2.26-2.27) in Proposition 2.11, we have

$$
(\sharp)=-R\left(J(U) \mathcal{U}, U_{x}\right) Y-\frac{1}{2}\left(B_{2}(U)+B_{3}(U)\right) Y-J(U) R\left(U_{x}, \partial_{x}(N(U)) Y\right) U_{x} .
$$

Substituting (2.35) and (2.36) into (2.34), we obtain the part of the summation of the first four terms of the right hand side of (2.32).

Remark 2.13. For any $Y:[-T, T] \times \mathbb{T} \rightarrow \mathbb{R}^{d}$, since $N(U) Y=\sum_{k}\left(Y, \nu_{k}(U)\right) \nu_{k}(U)$ and $A(U)\left(Y, U_{x}\right)=A(U)\left(P(U) Y, U_{x}\right)$, we find

$$
\begin{aligned}
& \partial_{x}(N(U)) Y\left(=-\partial_{x}(P(U)) Y\right) \\
& =\sum_{\ell}\left(Y, D_{\ell}(U) U_{x}\right) \nu_{\ell}(U)+\sum_{\ell}\left(Y, \nu_{\ell}(U)\right) D_{\ell}(U) U_{x} \\
& =A(U)\left(Y, U_{x}\right)+\sum_{\ell}\left(N(U) Y, D_{\ell}(U) U_{x}\right) \nu_{\ell}(U)+\sum_{\ell}\left(Y, \nu_{\ell}(U)\right) D_{\ell}(U) U_{x} .
\end{aligned}
$$

Definition 2.14. We define operators $S_{+}(U)$ and $S_{-}(U)$ by

$$
S_{ \pm}(U) Y:=\sum_{k}\left(U_{x}, D_{k}(U) U_{x}\right) P(U)\left(J(U) D_{k}(U) \pm D_{k}(U) J(U)\right) P(U) Y
$$

for any $Y:[-T, T] \times \mathbb{T} \rightarrow \mathbb{R}^{d}$.

By (2.3) and the symmetry of $D_{k}(U)$, it is immediate to see $\left(S_{ \pm}(U) Y_{1}, Y_{2}\right)=\mp\left(Y_{1}, S_{ \pm} Y_{2}\right)$ for any $Y_{1}, Y_{2}:[-T, T] \times \mathbb{T} \rightarrow \mathbb{R}^{d}$. In our proof of Theorem 1.3, the skew-symmetry of $S_{+}$ and the symmetry of $S_{-}$will play the crucial role. Furthermore, let us here look at the structure of $S_{ \pm}(U)$ in terms of $R, T(U)$, and the adjoint $(T(U))^{*}$ which is obviously given by

$$
(T(U))^{*}=\sum_{k}\left(\cdot, J(U) D_{k}(U) U_{x}\right) P(U) D_{k}(U) U_{x} .
$$

The observation will make the structure of the equation satisfied by $\mathcal{W}$ clear. For this purpose, let $Y:[-T, T] \times \mathbb{T} \rightarrow \mathbb{R}^{d}$ be any given. Applying (2.16) with $Y_{1}=Y, Y_{2}=Y_{3}=U_{x}$, and using (2.21) and the symmetry of $D_{k}(U)$, we have

$$
\begin{aligned}
& \sum_{k}\left(U_{x}, D_{k}(U) U_{x}\right) P(U) J(U) D_{k}(U) P(U) Y \\
& =J(U) \sum_{k}\left(U_{x}, D_{k}(U) U_{x}\right) P(U) D_{k}(U) P(U) Y \\
& =J(U) R\left(Y, U_{x}\right) U_{x}+J(U) \sum_{k}\left(U_{x}, D_{k}(U) P(U) Y\right) P(U) D_{k}(U) U_{x} \\
& =R\left(Y, U_{x}\right) J(U) U_{x}+\sum_{k}\left(P(U) Y, D_{k}(U) U_{x}\right) J(U) D_{k}(U) U_{x} \\
& =R\left(Y, U_{x}\right) J(U) U_{x}+T(U) Y .
\end{aligned}
$$

In the same way as above, applying (2.16) with $Y_{1}=J(U) Y, Y_{2}=Y_{3}=U_{x}$, and using (2.23), the symmetry of $D_{k}(U)$ and (2.3), we deduce

$$
\sum_{k}\left(U_{x}, D_{k}(U) U_{x}\right) P(U) D_{k}(U) J(U) P(U) Y
$$




$$
\begin{aligned}
& =\sum_{k}\left(U_{x}, D_{k}(U) U_{x}\right) P(U) D_{k}(U) P(U) J(U) Y \\
& =R\left(J(U) Y, U_{x}\right) U_{x}+\sum_{k}\left(U_{x}, D_{k}(U) J(U) Y\right) P(U) D_{k}(U) U_{x} \\
& =-R\left(Y, J(U) U_{x}\right) U_{x}-\sum_{k}\left(Y, J(U) D_{k}(U) U_{x}\right) P(U) D_{k}(U) U_{x} \\
& =-R\left(Y, J(U) U_{x}\right) U_{x}-(T(U))^{*} Y .
\end{aligned}
$$

Combining (2.40) and (2.41), and using (2.18) and (2.20), we find

$$
\begin{aligned}
S_{+}(U) & =T(U)-(T(U))^{*}+R\left(\cdot, U_{x}\right) J(U) U_{x}-R\left(\cdot, J(U) U_{x}\right) U_{x} \\
& =T(U)-(T(U))^{*}+R\left(J(U) U_{x}, U_{x}\right), \\
S_{-}(U) & =T(U)+(T(U))^{*}+R\left(\cdot, U_{x}\right) J(U) U_{x}+R\left(\cdot, J(U) U_{x}\right) U_{x} .
\end{aligned}
$$

It is obvious to see both $T(U)-(T(U))^{*}$ and $R\left(J(U) U_{x}, U_{x}\right)$ are skew-symmetric and both $T(U)+(T(U))^{*}$ and $R\left(\cdot, U_{x}\right) J(U) U_{x}+R\left(\cdot, J(U) U_{x}\right) U_{x}$ are symmetric. We can observe the skew-symmetry of $S_{+}(U)$ and symmetry of $S_{-}(U)$ also from them.

\section{EQUATIONS SATISFIED B Y $\mathcal{U}$ AND $\mathcal{W}$, AND THE ENERGY ESTIMATE IN $L^{2}$}

Let $u, v$ be solutions to (1.4)-(1.5) constructed in Theorem 1.2. Then $u$ and $v$ satisfy $u_{x}, v_{x} \in L^{\infty}\left(-T, T ; H^{5}(\mathbb{T} ; T N)\right) \cap C\left([-T, T] ; H^{4}(\mathbb{T} ; T N)\right)$. We fix $w$ as an isometric embedding of $(N, J, h)$ into some Euclidean space $\mathbb{R}^{d}$. We set $U:=w \circ u, V:=w \circ v$, $Z:=U-V, \mathcal{U}:=d w_{u}\left(\nabla_{x} u_{x}\right), \mathcal{V}:=d w_{v}\left(\nabla_{x} v_{x}\right)$, and $\mathcal{W}:=\mathcal{U}-\mathcal{V}$. The goal of this section is to derive the equation satisfied by $\mathcal{W}$ and the classical estimate for $\mathcal{W}$ in $L^{2}\left(\mathbb{T} ; \mathbb{R}^{d}\right)$ permitting loss of derivatives.

Before starting them, some comments are in order: The equivalence between $u_{x}, v_{x} \in$ $L^{\infty}\left(-T, T ; H^{5}(\mathbb{T} ; T N)\right) \cap C\left([-T, T] ; H^{4}(\mathbb{T} ; T N)\right)$ and $U_{x}, V_{x} \in L^{\infty}\left(-T, T ; H^{5}\left(\mathbb{T} ; \mathbb{R}^{d}\right)\right) \cap$ $C\left([-T, T] ; H^{4}\left(\mathbb{T} ; \mathbb{R}^{d}\right)\right)$ follows from the Gagliardo-Nirenberg inequality. Therefore we see

$$
\mathcal{U}, \mathcal{V}, \mathcal{W} \in L^{\infty}\left(-T, T ; H^{4}\left(\mathbb{T} ; \mathbb{R}^{d}\right)\right) \cap C\left([-T, T] ; H^{3}\left(\mathbb{T} ; \mathbb{R}^{d}\right)\right)
$$

Particularly from the Sobolev embedding $H^{1}\left(\mathbb{T} ; \mathbb{R}^{d}\right) \subset C\left(\mathbb{T} ; \mathbb{R}^{d}\right)$, we see $\partial_{x}^{\ell} U_{x}, \partial_{x}^{\ell} V_{x} \in$ $C\left([-T, T] \times \mathbb{T} ; \mathbb{R}^{d}\right)$ for $\ell=0,1, \ldots, 3$ and $\partial_{x}^{\ell} \mathcal{U}, \partial_{x}^{\ell} \mathcal{V} \in C\left([-T, T] \times \mathbb{T} ; \mathbb{R}^{d}\right)$ for $\ell=$ $0,1, \ldots, 2$. They will be used below without any comments. In addition, we will sometimes write $d w$ instead of $d w_{u}$ or $d w_{v}$ below for simplicity.

3.1. Equation satisfied by $\mathcal{U}$. In this subsection, we compute the equation satisfied by $\mathcal{U}$. The goal is to derive the following:

Proposition 3.1. Under the setting as above, we have

$$
\partial_{t} \mathcal{U}=L(U) \mathcal{U}+\sum_{k} \mathcal{O}\left(\left|\partial_{x}^{2} \mathcal{U}\right|+\left|\partial_{x} \mathcal{U}\right|\right) \nu_{k}(U)+\mathcal{O}\left(|U|+\left|U_{x}\right|+|\mathcal{U}|\right)
$$

where $L(U)$ is the partial differential operator which is given by

$$
\begin{aligned}
L(U) Y= & a \partial_{x}^{2}\left\{J(U) \partial_{x}^{2} Y\right\}+2 a A(U)\left(J(U) \partial_{x}^{3} Y, U_{x}\right)+\lambda \partial_{x}\left\{J(U) \partial_{x} Y\right\} \\
& +(-a+b) R\left(\partial_{x}^{2} Y, J(U) U_{x}\right) U_{x}+(-a+b+c) \partial_{x}\left\{R\left(J(U) U_{x}, U_{x}\right) \partial_{x} Y\right\} \\
& +\left(-\frac{a}{2}-\frac{b}{2}+3 c\right) R\left(J(U) \mathcal{U}, U_{x}\right) \partial_{x} Y+\sum_{i=1}^{3} c_{i} B_{i}(U) \partial_{x} Y
\end{aligned}
$$




$$
\begin{aligned}
& +a \partial_{x}\left\{S_{+}(U) \partial_{x} Y\right\}+a \partial_{x}\left(S_{-}(U)\right) \partial_{x} Y \\
& +2 a \sum_{k}\left(\mathcal{U}, D_{k}(U) U_{x}\right) J(U) D_{k}(U) \partial_{x} Y
\end{aligned}
$$

for any $Y:[-T, T] \times \mathbb{T} \rightarrow \mathbb{R}^{d}$. Here, $c_{1}, c_{2}, c_{3} \in \mathbb{R}$ are constants depending on $a, b, c$, and $B_{i}(U)$ for $i=1,2,3$ have been defined in Definition 2.9. and $S_{+}(U)$ and $S_{-}(U)$ have been defined in Definition 2.14 or 2.42)-2.43.

To prove this, lengthy and delicate computations will be required. For readers who want to know how Proposition 3.1 is applied in advance, it is also recommendable to skip to Subsection 3.2 .

Proof of Proposition 3.1. To begin with, we compute the equation satisfied by $U$. Using (1.4) and (2.17), we deduce

$$
\begin{aligned}
U_{t}= & d w\left(u_{t}\right) \\
= & a d w\left(\nabla_{x} J_{u} \nabla_{x}^{2} u_{x}\right)+\lambda d w\left(J_{u} \nabla_{x} u_{x}\right) \\
& +b d w\left(R^{N}\left(\nabla_{x} u_{x}, u_{x}\right) J_{u} u_{x}\right)+c d w\left(R^{N}\left(J_{u} u_{x}, u_{x}\right) \nabla_{x} u_{x}\right) \\
= & a d w\left(\nabla_{x} J_{u} \nabla_{x}^{2} u_{x}\right)+\lambda J(U) d w\left(\nabla_{x} u_{x}\right) \\
& +b R\left(d w\left(\nabla_{x} u_{x}\right), d w\left(u_{x}\right)\right) d w\left(J_{u} u_{x}\right) \\
& +c R\left(d w\left(J_{u} u_{x}\right), d w\left(u_{x}\right)\right) d w\left(\nabla_{x} u_{x}\right) \\
= & a d w\left(\nabla_{x} J_{u} \nabla_{x}^{2} u_{x}\right)+\lambda J(U) \mathcal{U}+b R\left(\mathcal{U}, U_{x}\right) J(U) U_{x}+c R\left(J(U) U_{x}, U_{x}\right) \mathcal{U} .
\end{aligned}
$$

In the computation, we also repeatedly use the fact $d w\left(J_{u} \Xi\right)=J(U) d w(\Xi)$ holds for any $\Xi \in \Gamma\left(u^{-1} T N\right)$. Note also that, for the first term of the right hand side, we use the fact $J_{u} \nabla_{x}^{3} u_{x}=\nabla_{x} J_{u} \nabla_{x}^{2} u_{x}$ which comes from the Kähler condition $\nabla^{N} J=0$. Furthermore, using (2.5) and $J(U) A(U)(\cdot, \cdot)=0$ which comes from (2.7), we see

$$
\begin{aligned}
d w\left(\nabla_{x}^{2} u_{x}\right) & =\partial_{x}\left\{d w\left(\nabla_{x} u_{x}\right)\right\}+A(U)\left(d w\left(\nabla_{x} u_{x}\right), U_{x}\right) \\
& =\partial_{x} \mathcal{U}+A(U)\left(\mathcal{U}, U_{x}\right), \\
J(U) d w\left(\nabla_{x}^{2} u_{x}\right) & =J(U) \partial_{x} \mathcal{U}+J(U) A(U)\left(\mathcal{U}, U_{x}\right)=J(U) \partial_{x} \mathcal{U}, \\
d w\left(\nabla_{x} J_{u} \nabla_{x}^{2} u_{x}\right) & =\partial_{x}\left\{d w\left(J_{u} \nabla_{x}^{2} u_{x}\right)\right\}+A(U)\left(d w\left(J_{u} \nabla_{x}^{2} u_{x}\right), U_{x}\right) \\
& =\partial_{x}\left\{J(U) d w\left(\nabla_{x}^{2} u_{x}\right)\right\}+A(U)\left(J(U) d w\left(\nabla_{x}^{2} u_{x}\right), U_{x}\right) \\
& =\partial_{x}\left\{J(U) \partial_{x} \mathcal{U}\right\}+A(U)\left(J(U) \partial_{x} \mathcal{U}, U_{x}\right) .
\end{aligned}
$$

Substituting (3.6) into (3.3), we have

$$
U_{t}=a \partial_{x}\left\{J(U) \partial_{x} \mathcal{U}\right\}+a A(U)\left(J(U) \partial_{x} \mathcal{U}, U_{x}\right)+\mathcal{O}\left(|U|+\left|U_{x}\right|+|\mathcal{U}|\right) .
$$

On the other hand, we notice $d w\left(\nabla_{x} J_{u} \nabla_{x}^{2} u_{x}\right)=d w\left(J_{u} \nabla_{x}^{3} u_{x}\right)=J(U) d w\left(\nabla_{x}^{3} u_{x}\right)$ also follows from the Kähler condition $\nabla^{N} J=0$. Using (2.5) and (3.4), we obtain

$$
\begin{aligned}
d w\left(\nabla_{x}^{3} u_{x}\right)= & \partial_{x}\left\{d w\left(\partial_{x}^{2} u_{x}\right)\right\}+A(U)\left(d w\left(\partial_{x}^{2} u_{x}\right), U_{x}\right) \\
= & \partial_{x}^{2} \mathcal{U}+\partial_{x}\left\{A(U)\left(\mathcal{U}, U_{x}\right)\right\}+A(U)\left(\partial_{x} \mathcal{U}+A(U)\left(\mathcal{U}, U_{x}\right), U_{x}\right) \\
= & \partial_{x}^{2} \mathcal{U}+\partial_{x}(A(U))\left(\mathcal{U}, U_{x}\right)+2 A(U)\left(\partial_{x} \mathcal{U}, U_{x}\right) \\
& +A(U)\left(\mathcal{U}, U_{x x}\right)+A(U)\left(A(U)\left(\mathcal{U}, U_{x}\right), U_{x}\right) .
\end{aligned}
$$


Hence, using $J(U) A(U)(\cdot, \cdot)=0$ again, we get

$$
d w\left(\nabla_{x} J_{u} \nabla_{x}^{2} u_{x}\right)=J(U) \partial_{x}^{2} \mathcal{U}+J(U) \partial_{x}(A(U))\left(\mathcal{U}, U_{x}\right) .
$$

Therefore, we notice (3.7) can be rewritten as

$$
U_{t}=a J(U) \partial_{x}^{2} \mathcal{U}+\mathcal{O}\left(|U|+\left|U_{x}\right|+|\mathcal{U}|\right)
$$

Next, we compute the equation satisfied by $\mathcal{U}$. From (1.4), (2.17), and (2.6), it follows that

$$
\begin{aligned}
\partial_{t} \mathcal{U} & =\partial_{t}\left(d w\left(\nabla_{x} u_{x}\right)\right) \\
& =d w\left(\nabla_{t} \nabla_{x} u_{x}\right)-A(U)\left(d w\left(\nabla_{x} u_{x}\right), U_{t}\right) \\
& =d w\left(\nabla_{x}^{2} u_{t}+R^{N}\left(u_{t}, u_{x}\right) u_{x}\right)-A(U)\left(\mathcal{U}, U_{t}\right) \\
& =d w\left(\nabla_{x}^{2} u_{t}\right)+R\left(U_{t}, U_{x}\right) U_{x}-A(U)\left(\mathcal{U}, U_{t}\right) \\
& =a d w\left(\nabla_{x}^{2} J_{u} \nabla_{x}^{3} u_{x}\right)+\lambda d w\left(\nabla_{x}^{2} J_{u} \nabla_{x} u_{x}\right)+F_{R}+R\left(U_{t}, U_{x}\right) U_{x}-A(U)\left(\mathcal{U}, U_{t}\right), \\
F_{R} & :=d w\left(b \nabla_{x}^{2}\left\{R^{N}\left(\nabla_{x} u_{x}, u_{x}\right) J_{u} u_{x}\right\}+c \nabla_{x}^{2}\left\{R^{N}\left(J_{u} u_{x}, u_{x}\right) \nabla_{x} u_{x}\right\}\right) .
\end{aligned}
$$

We compute each term of the right hand side of (3.11). Starting from substituting (3.10) and using (2.23), we have

$$
\begin{aligned}
R\left(U_{t}, U_{x}\right) U_{x} & =a R\left(J(U) \partial_{x}^{2} \mathcal{U}, U_{x}\right) U_{x}+\mathcal{O}\left(|U|+\left|U_{x}\right|+|\mathcal{U}|\right) \\
& =-a R\left(\partial_{x}^{2} \mathcal{U}, J(U) U_{x}\right) U_{x}+\mathcal{O}\left(|U|+\left|U_{x}\right|+|\mathcal{U}|\right), \\
A(U)\left(\mathcal{U}, U_{t}\right) & =a A(U)\left(\mathcal{U}, J(U) \partial_{x}^{2} \mathcal{U}\right)+\mathcal{O}\left(|U|+\left|U_{x}\right|+|\mathcal{U}|\right) .
\end{aligned}
$$

Using (3.6) and the Kähler condition $\nabla^{N} J=0$, we immediately see

$$
\lambda d w\left(\nabla_{x}^{2} J_{u} \nabla_{x} u_{x}\right)=\lambda \partial_{x}\left\{J(U) \partial_{x} \mathcal{U}\right\}+\lambda A(U)\left(J(U) \partial_{x} \mathcal{U}, U_{x}\right) .
$$

Concerning the term $F_{R}$, we compute using Proposition 2.4. Indeed, using (i) and (vii) in Proposition 2.4, we deduce

$$
\begin{aligned}
F_{R 1}:= & \nabla_{x}^{2}\left\{R^{N}\left(\nabla_{x} u_{x}, u_{x}\right) J_{u} u_{x}\right\} \\
= & R^{N}\left(\nabla_{x}^{3} u_{x}, u_{x}\right) J_{u} u_{x}+R^{N}\left(\nabla_{x} u_{x}, \nabla_{x}^{2} u_{x}\right) J_{u} u_{x} \\
& +R^{N}\left(\nabla_{x} u_{x}, u_{x}\right) J_{u} \nabla_{x}^{2} u_{x}+2 R^{N}\left(\nabla_{x}^{2} u_{x}, \nabla_{x} u_{x}\right) J_{u} u_{x} \\
& +2 R^{N}\left(\nabla_{x}^{2} u_{x}, u_{x}\right) J_{u} \nabla_{x} u_{x}+2 R^{N}\left(\nabla_{x} u_{x}, \nabla_{x} u_{x}\right) J_{u} \nabla_{x} u_{x} \\
= & R^{N}\left(\nabla_{x}^{3} u_{x}, u_{x}\right) J_{u} u_{x}+R^{N}\left(\nabla_{x}^{2} u_{x}, \nabla_{x} u_{x}\right) J_{u} u_{x} \\
& +2 R^{N}\left(\nabla_{x}^{2} u_{x}, u_{x}\right) J_{u} \nabla_{x} u_{x}+R^{N}\left(\nabla_{x} u_{x}, u_{x}\right) J_{u} \nabla_{x}^{2} u_{x} .
\end{aligned}
$$

Then, using (iii) in Proposition 2.4, we have

$$
\begin{aligned}
F_{R 1}= & -R^{N}\left(u_{x}, J_{u} u_{x}\right) \nabla_{x}^{3} u_{x}-R^{N}\left(J_{u} u_{x}, \nabla_{x}^{3} u_{x}\right) u_{x}-R^{N}\left(\nabla_{x} u_{x}, J_{u} u_{x}\right) \nabla_{x}^{2} u_{x} \\
& -R^{N}\left(J_{u} u_{x}, \nabla_{x}^{2} u_{x}\right) \nabla_{x} u_{x}-2 R^{N}\left(u_{x}, J_{u} \nabla_{x} u_{x}\right) \nabla_{x}^{2} u_{x} \\
& -2 R^{N}\left(J_{u} \nabla_{x} u_{x}, \nabla_{x}^{2} u_{x}\right) u_{x}-R^{N}\left(u_{x}, J_{u} \nabla_{x}^{2} u_{x}\right) \nabla_{x} u_{x}-R^{N}\left(J_{u} \nabla_{x}^{2} u_{x}, \nabla_{x} u_{x}\right) u_{x} .
\end{aligned}
$$

Furthermore, using (i) and (vi) in Proposition 2.4, we get

$$
\begin{aligned}
F_{R 1}= & R^{N}\left(\nabla_{x}^{3} u_{x}, J_{u} u_{x}\right) u_{x}+R^{N}\left(J_{u} u_{x}, u_{x}\right) \nabla_{x}^{3} u_{x} \\
& -3 R^{N}\left(\nabla_{x} u_{x}, J_{u} u_{x}\right) \nabla_{x}^{2} u_{x}+3 R^{N}\left(\nabla_{x}^{2} u_{x}, J_{u} \nabla_{x} u_{x}\right) u_{x} .
\end{aligned}
$$


In the same way, using (vii) in Proposition 2.4, we deduce

$$
\begin{aligned}
F_{R 2}:= & \nabla_{x}^{2}\left\{R^{N}\left(J_{u} u_{x}, u_{x}\right) \nabla_{x} u_{x}\right\} \\
= & R^{N}\left(J_{u} \nabla_{x}^{2} u_{x}, u_{x}\right) \nabla_{x} u_{x}+R^{N}\left(J_{u} u_{x}, \nabla_{x}^{2} u_{x}\right) \nabla_{x} u_{x} \\
& +2 R^{N}\left(J_{u} \nabla_{x} u_{x}, \nabla_{x} u_{x}\right) \nabla_{x} u_{x}+2 R^{N}\left(J_{u} \nabla_{x} u_{x}, u_{x}\right) \nabla_{x}^{2} u_{x} \\
& +2 R^{N}\left(J_{u} u_{x}, \nabla_{x} u_{x}\right) \nabla_{x}^{2} u_{x}+R^{N}\left(J_{u} u_{x}, u_{x}\right) \nabla_{x}^{3} u_{x} .
\end{aligned}
$$

Then, using (i) and (vi) in Proposition 2.4, we have

$$
\begin{aligned}
F_{R 2}= & R^{N}\left(J_{u} u_{x}, u_{x}\right) \nabla_{x}^{3} u_{x}-4 R^{N}\left(\nabla_{x} u_{x}, J_{u} u_{x}\right) \nabla_{x}^{2} u_{x} \\
& -2 R^{N}\left(\nabla_{x}^{2} u_{x}, J_{u} u_{x}\right) \nabla_{x} u_{x}+2 R^{N}\left(J_{u} \nabla_{x} u_{x}, \nabla_{x} u_{x}\right) \nabla_{x} u_{x} .
\end{aligned}
$$

Substituting them into $F_{R}=d w\left(b F_{R 1}+c F_{R 2}\right)$, we deduce

$$
\begin{aligned}
F_{R}= & d w\left(b R^{N}\left(\nabla_{x}^{3} u_{x}, J_{u} u_{x}\right) u_{x}+(b+c) R^{N}\left(J_{u} u_{x}, u_{x}\right) \nabla_{x}^{3} u_{x}\right. \\
& -(3 b+4 c) R^{N}\left(\nabla_{x} u_{x}, J_{u} u_{x}\right) \nabla_{x}^{2} u_{x}+3 b R^{N}\left(\nabla_{x}^{2} u_{x}, J_{u} \nabla_{x} u_{x}\right) u_{x} \\
& \left.-2 c R^{N}\left(\nabla_{x}^{2} u_{x}, J_{u} u_{x}\right) \nabla_{x} u_{x}+2 c R^{N}\left(J_{u} \nabla_{x} u_{x}, \nabla_{x} u_{x}\right) \nabla_{x} u_{x}\right) \\
= & b R\left(d w\left(\nabla_{x}^{3} u_{x}\right), J(U) U_{x}\right) U_{x}+(b+c) R\left(J(U) U_{x}, U_{x}\right) d w\left(\nabla_{x}^{3} u_{x}\right) \\
& -(3 b+4 c) R\left(\mathcal{U}, J(U) U_{x}\right) d w\left(\nabla_{x}^{2} u_{x}\right)+3 b R\left(d w\left(\nabla_{x}^{2} u_{x}\right), J(U) \mathcal{U}\right) U_{x} \\
& -2 c R\left(d w\left(\nabla_{x}^{2} u_{x}\right), J(U) U_{x}\right) \mathcal{U}+2 c R(J(U) \mathcal{U}, \mathcal{U}) \mathcal{U} .
\end{aligned}
$$

Substituting (3.4) and (3.8) into above, and noting (2.15) holds, we obtain

$$
\begin{aligned}
F_{R}= & b R\left(\partial_{x}^{2} \mathcal{U}, J(U) U_{x}\right) U_{x}+(b+c) R\left(J(U) U_{x}, U_{x}\right) \partial_{x}^{2} \mathcal{U} \\
& +2 b R\left(A(U)\left(\partial_{x} \mathcal{U}, U_{x}\right), J(U) U_{x}\right) U_{x}+2(b+c) R\left(J(U) U_{x}, U_{x}\right) A(U)\left(\partial_{x} \mathcal{U}, U_{x}\right) \\
& -(3 b+4 c) R\left(\mathcal{U}, J(U) U_{x}\right) \partial_{x} \mathcal{U}+3 b R\left(\partial_{x} \mathcal{U}, J(U) \mathcal{U}\right) U_{x} \\
& -2 c R\left(\partial_{x} \mathcal{U}, J(U) U_{x}\right) \mathcal{U}+\mathcal{O}\left(|U|+\left|U_{x}\right|+|\mathcal{U}|\right) \\
= & b R\left(\partial_{x}^{2} \mathcal{U}, J(U) U_{x}\right) U_{x}+(b+c) R\left(J(U) U_{x}, U_{x}\right) \partial_{x}^{2} \mathcal{U} \\
& -(3 b+4 c) R\left(\mathcal{U}, J(U) U_{x}\right) \partial_{x} \mathcal{U}+3 b R\left(\partial_{x} \mathcal{U}, J(U) \mathcal{U}\right) U_{x} \\
& -2 c R\left(\partial_{x} \mathcal{U}, J(U) U_{x}\right) \mathcal{U}+\mathcal{O}\left(|U|+\left|U_{x}\right|+|\mathcal{U}|\right) .
\end{aligned}
$$

Furthermore, using (2.24) with $Y_{1}=J(U) U_{x}, Y_{2}=U_{x}, Y_{3}=\partial_{x} \mathcal{U}$, we have

$$
\begin{aligned}
\partial_{x} & \left\{R\left(J(U) U_{x}, U_{x}\right) \partial_{x} \mathcal{U}\right\} \\
= & R\left(\partial_{x}\left\{J(U) U_{x}\right\}, U_{x}\right) \partial_{x} \mathcal{U}+R\left(J(U) U_{x}, U_{x x}\right) \partial_{x} \mathcal{U} \\
& +R\left(J(U) U_{x}, U_{x}\right) \partial_{x}\left\{P(U) \partial_{x} \mathcal{U}\right\}-A(U)\left(R\left(J(U) U_{x}, U_{x}\right) \partial_{x} \mathcal{U}, U_{x}\right) .
\end{aligned}
$$

Hence, using $\partial_{x}\left\{P(U) \partial_{x} \mathcal{U}\right\}=P(U) \partial_{x}^{2} \mathcal{U}+\partial_{x}(P(U)) \partial_{x} \mathcal{U}$ and (2.14), we obtain

$$
\begin{aligned}
& R\left(J(U) U_{x}, U_{x}\right) \partial_{x}^{2} \mathcal{U} \\
& =\partial_{x}\left\{R\left(J(U) U_{x}, U_{x}\right) \partial_{x} \mathcal{U}\right\}-R\left(\partial_{x}\left\{J(U) U_{x}\right\}, U_{x}\right) \partial_{x} \mathcal{U} \\
& \quad-R\left(J(U) U_{x}, U_{x x}\right) \partial_{x} \mathcal{U}-R\left(J(U) U_{x}, U_{x}\right) \partial_{x}(P(U)) \partial_{x} \mathcal{U} \\
& \quad+A(U)\left(R\left(J(U) U_{x}, U_{x}\right) \partial_{x} \mathcal{U}, U_{x}\right) .
\end{aligned}
$$

Here, from 2.8 and (2.7), we see

$$
\partial_{x}\left\{J(U) U_{x}\right\}=\partial_{x}(J(U)) U_{x}+J(U) U_{x x}=-A(U)\left(J(U) U_{x}, U_{x}\right)+J(U) \mathcal{U} .
$$


In addition, recalling (2.37) and using $\left(\partial_{x} \mathcal{U}, \nu_{k}(U)\right)=-\left(\mathcal{U}, D_{k}(U) U_{x}\right)$ which comes from $\mathcal{U} \perp \nu_{k}(U)$, we have

$$
\begin{aligned}
\partial_{x}(P(U)) \partial_{x} \mathcal{U}= & -A(U)\left(\partial_{x} \mathcal{U}, U_{x}\right)-\sum_{k}\left(N(U) \partial_{x} \mathcal{U}, D_{k}(U) U_{x}\right) \nu_{k}(U) \\
& -\sum_{k}\left(\partial_{x} \mathcal{U}, \nu_{k}(U)\right) D_{k}(U) U_{x} \\
= & -A(U)\left(\partial_{x} \mathcal{U}, U_{x}\right)+\mathcal{O}\left(|U|+\left|U_{x}\right|+|\mathcal{U}|\right) .
\end{aligned}
$$

Substituting (3.16) and (3.17), and then using $U_{x x}+A(U)\left(U_{x}, U_{x}\right)=\mathcal{U}$, (2.23), and 2.15), we deduce

$$
\begin{aligned}
& R\left(J(U) U_{x}, U_{x}\right) \partial_{x}^{2} \mathcal{U} \\
&= \partial_{x}\left\{R\left(J(U) U_{x}, U_{x}\right) \partial_{x} \mathcal{U}\right\}-R\left(J(U) \mathcal{U}, U_{x}\right) \partial_{x} \mathcal{U} \\
& \quad-R\left(J(U) U_{x}, U_{x x}\right) \partial_{x} \mathcal{U}+R\left(J(U) U_{x}, U_{x}\right) A(U)\left(\partial_{x} \mathcal{U}, U_{x}\right) \\
& \quad+A(U)\left(R\left(J(U) U_{x}, U_{x}\right) \partial_{x} \mathcal{U}, U_{x}\right)+\mathcal{O}\left(|U|+\left|U_{x}\right|+|\mathcal{U}|\right) \\
&=\partial_{x}\left\{R\left(J(U) U_{x}, U_{x}\right) \partial_{x} \mathcal{U}\right\}-2 R\left(J(U) \mathcal{U}, U_{x}\right) \partial_{x} \mathcal{U} \\
& \quad+A(U)\left(R\left(J(U) U_{x}, U_{x}\right) \partial_{x} \mathcal{U}, U_{x}\right)+\mathcal{O}\left(|U|+\left|U_{x}\right|+|\mathcal{U}|\right) .
\end{aligned}
$$

Substituting (3.18) into (3.15) and then using (2.23), we obtain

$$
\begin{aligned}
F_{R}= & b R\left(\partial_{x}^{2} \mathcal{U}, J(U) U_{x}\right) U_{x}+(b+c) \partial_{x}\left\{R\left(J(U) U_{x}, U_{x}\right) \partial_{x} \mathcal{U}\right\} \\
& +(b+2 c) R\left(J(U) \mathcal{U}, U_{x}\right) \partial_{x} \mathcal{U}+3 b R\left(\partial_{x} \mathcal{U}, J(U) \mathcal{U}\right) U_{x}-2 c R\left(\partial_{x} \mathcal{U}, J(U) U_{x}\right) \mathcal{U} \\
& +(b+c) A(U)\left(R\left(J(U) U_{x}, U_{x}\right) \partial_{x} \mathcal{U}, U_{x}\right)+\mathcal{O}\left(|U|+\left|U_{x}\right|+|\mathcal{U}|\right) .
\end{aligned}
$$

We observe the structure of $F_{R}$ in more detail by using (2.26) and 2.27) in Proposition 2.11. Indeed, using 2.20) with $Y_{1}=\partial_{x} \mathcal{U}, Y_{2}=J(U) \mathcal{U}, Y_{3}=U_{x}$ and (2.18), and then using 2.27) with $Y=\partial_{x} \mathcal{U}$, we deduce

$$
\begin{aligned}
& R\left(\partial_{x} \mathcal{U}, J(U) \mathcal{U}\right) U_{x} \\
& =-R\left(J(U) \mathcal{U}, U_{x}\right) \partial_{x} \mathcal{U}-R\left(U_{x}, \partial_{x} \mathcal{U}\right) J(U) \mathcal{U} \\
& =-R\left(J(U) \mathcal{U}, U_{x}\right) \partial_{x} \mathcal{U}+R\left(\partial_{x} \mathcal{U}, U_{x}\right) J(U) \mathcal{U} \\
& =-\frac{1}{2} R\left(J(U) \mathcal{U}, U_{x}\right) \partial_{x} \mathcal{U}-\left(\frac{B_{1}(U)}{2}-\frac{B_{2}(U)}{4}-\frac{B_{3}(U)}{4}\right) \partial_{x} \mathcal{U} .
\end{aligned}
$$

In the same way, using 2.26, we can obtain

$$
\begin{aligned}
& R\left(\partial_{x} \mathcal{U}, J(U) U_{x}\right) \mathcal{U} \\
& =-\frac{1}{2} R\left(J(U) \mathcal{U}, U_{x}\right) \partial_{x} \mathcal{U}+\left(\frac{B_{1}(U)}{2}+\frac{B_{2}(U)}{4}+\frac{B_{3}(U)}{4}\right) \partial_{x} \mathcal{U} .
\end{aligned}
$$

Substituting (3.20) and (3.21), we have

$$
\begin{aligned}
F_{R}= & b R\left(\partial_{x}^{2} \mathcal{U}, J(U) U_{x}\right) U_{x}+(b+c) \partial_{x}\left\{R\left(J(U) U_{x}, U_{x}\right) \partial_{x} \mathcal{U}\right\} \\
& +\left(-\frac{b}{2}+3 c\right) R\left(J(U) \mathcal{U}, U_{x}\right) \partial_{x} \mathcal{U}+\sum_{i=1}^{3} b_{i} B_{i}(U) \partial_{x} \mathcal{U} \\
& +(b+c) A(U)\left(R\left(J(U) U_{x}, U_{x}\right) \partial_{x} \mathcal{U}, U_{x}\right)+\mathcal{O}\left(|U|+\left|U_{x}\right|+|\mathcal{U}|\right),
\end{aligned}
$$


where $b_{1}=-\frac{3 b}{2}-c, b_{2}=b_{3}=\frac{3 b}{4}-\frac{c}{2}$, though the constants are now not important. Substituting (3.12), 3.13), 3.14) and (3.22) into (3.11), we arrive at the following.

$$
\begin{aligned}
\partial_{t} \mathcal{U}= & a d w\left(\nabla_{x}^{2} J_{u} \nabla_{x}^{3} u_{x}\right)+\lambda \partial_{x}\left\{J(U) \partial_{x} \mathcal{U}\right\} \\
& +(-a+b) R\left(\partial_{x}^{2} \mathcal{U}, J(U) U_{x}\right) U_{x}+(b+c) \partial_{x}\left\{R\left(J(U) U_{x}, U_{x}\right) \partial_{x} \mathcal{U}\right\} \\
& +\left(-\frac{b}{2}+3 c\right) R\left(J(U) \mathcal{U}, U_{x}\right) \partial_{x} \mathcal{U}+\sum_{i=1}^{3} b_{i} B_{i}(U) \partial_{x} \mathcal{U} \\
& +r\left(U, U_{x}, \mathcal{U}, \partial_{x} \mathcal{U}, \partial_{x}^{2} \mathcal{U}\right)+\mathcal{O}\left(|U|+\left|U_{x}\right|+|\mathcal{U}|\right),
\end{aligned}
$$

where

$$
\begin{aligned}
r\left(U, U_{x}, \mathcal{U}, \partial_{x} \mathcal{U}, \partial_{x}^{2} \mathcal{U}\right)= & \lambda A(U)\left(J(U) \partial_{x} \mathcal{U}, U_{x}\right)-a A(U)\left(\mathcal{U}, J(U) \partial_{x}^{2} \mathcal{U}\right) \\
& +(b+c) A(U)\left(R\left(J(U) U_{x}, U_{x}\right) \partial_{x} \mathcal{U}, U_{x}\right) \\
= & \sum_{k} \mathcal{O}\left(\left|\partial_{x}^{2} \mathcal{U}\right|+\left|\partial_{x} \mathcal{U}\right|\right) \nu_{k}(U)
\end{aligned}
$$

Let us now move on to the computation of the first term of the right hand side of (3.23). We set $I(U):=d w\left(\nabla_{x}^{2} J_{u} \nabla_{x}^{3} u_{x}\right)$. Starting from using (2.5) twice,

$$
\begin{aligned}
I(U)= & \partial_{x}\left\{d w\left(\nabla_{x} J_{u} \nabla_{x}^{3} u_{x}\right)\right\}+A(U)\left(d w\left(\nabla_{x} J_{u} \nabla_{x}^{3} u_{x}\right), U_{x}\right) \\
= & \partial_{x}\left\{\partial_{x}\left\{d w\left(J_{u} \nabla_{x}^{3} u_{x}\right)\right\}+A(U)\left(d w\left(J_{u} \nabla_{x}^{3} u_{x}\right), U_{x}\right)\right\} \\
& +A(U)\left(\partial_{x}\left\{d w\left(J_{u} \nabla_{x}^{3} u_{x}\right)\right\}+A(U)\left(d w\left(J_{u} \nabla_{x}^{3} u_{x}\right), U_{x}\right), U_{x}\right) .
\end{aligned}
$$

We have already obtained $d w\left(J_{u} \nabla_{x}^{3} u_{x}\right)=J(U) \partial_{x}^{2} \mathcal{U}+J(U) \partial_{x}(A(U))\left(\mathcal{U}, U_{x}\right)$ by (3.9). Substituting this into the above, we have

$$
\begin{aligned}
I(U)= & \partial_{x}^{2}\left\{J(U) \partial_{x}^{2} \mathcal{U}\right\}+\partial_{x}^{2}\left\{J(U) \partial_{x}(A(U))\left(\mathcal{U}, U_{x}\right)\right\} \\
& +\partial_{x}\left\{A(U)\left(J(U) \partial_{x}^{2} \mathcal{U}+J(U) \partial_{x}(A(U))\left(\mathcal{U}, U_{x}\right), U_{x}\right)\right\} \\
& +A(U)\left(\partial_{x}\left\{J(U) \partial_{x}^{2} \mathcal{U}+J(U) \partial_{x}(A(U))\left(\mathcal{U}, U_{x}\right)\right\}, U_{x}\right) \\
& +A(U)\left(A(U)\left(J(U) \partial_{x}^{2} \mathcal{U}+J(U) \partial_{x}(A(U))\left(\mathcal{U}, U_{x}\right), U_{x}\right), U_{x}\right) .
\end{aligned}
$$

By a simple computation, we see

$$
\begin{aligned}
I(U)= & \partial_{x}^{2}\left\{J(U) \partial_{x}^{2} \mathcal{U}\right\}+2 A(U)\left(J(U) \partial_{x}^{3} \mathcal{U}, U_{x}\right) \\
& +J(U) \partial_{x}(A(U))\left(\partial_{x}^{2} \mathcal{U}, U_{x}\right)+\partial_{x}(A(U))\left(J(U) \partial_{x}^{2} \mathcal{U}, U_{x}\right) \\
& +2 J(U) \partial_{x}(A(U))\left(\partial_{x} \mathcal{U}, U_{x x}\right)+2 \partial_{x}(J(U)) \partial_{x}(A(U))\left(\partial_{x} \mathcal{U}, U_{x}\right) \\
& +2 J(U) \partial_{x}^{2}(A(U))\left(\partial_{x} \mathcal{U}, U_{x}\right)+J(U) \partial_{x}(A(U))\left(\mathcal{U}, U_{x x x}\right) \\
& +J(U) \partial_{x}^{3}(A(U))\left(\mathcal{U}, U_{x}\right) \\
& +r_{1}\left(U, U_{x}, \mathcal{U}, \partial_{x} \mathcal{U}, \partial_{x}^{2} \mathcal{U}\right)+\mathcal{O}\left(|U|+\left|U_{x}\right|+|\mathcal{U}|\right),
\end{aligned}
$$

where

$$
\begin{aligned}
r_{1}\left(U, U_{x}, \mathcal{U}, \partial_{x} \mathcal{U}, \partial_{x}^{2} \mathcal{U}\right) & =A(U)\left(\mathcal{O}\left(\left|\partial_{x}^{2} \mathcal{U}\right|+\left|\partial_{x} \mathcal{U}\right|\right),\left|U_{x x}\right|+\left|U_{x}\right|\right) \\
& =\sum_{k} \mathcal{O}\left(\left|\partial_{x}^{2} \mathcal{U}\right|+\left|\partial_{x} \mathcal{U}\right|\right) \nu_{k}(U)
\end{aligned}
$$


Thus, we can rewrite as

$$
\begin{aligned}
I(U)= & \partial_{x}^{2}\left\{J(U) \partial_{x}^{2} \mathcal{U}\right\}+2 A(U)\left(J(U) \partial_{x}^{3} \mathcal{U}, U_{x}\right) \\
& +G_{1}^{I I}+G_{2}^{I I}+2 G_{1}^{I}+2 G_{2}^{I}+2 G_{3}^{I}+G_{4}^{I}+G_{5}^{I} \\
& +\sum_{k} \mathcal{O}\left(\left|\partial_{x}^{2} \mathcal{U}\right|+\left|\partial_{x} \mathcal{U}\right|\right) \nu_{k}(U)+\mathcal{O}\left(|U|+\left|U_{x}\right|+|\mathcal{U}|\right),
\end{aligned}
$$

where

$$
\begin{array}{ll}
G_{1}^{I I}=J(U) \partial_{x}(A(U))\left(\partial_{x}^{2} \mathcal{U}, U_{x}\right), & G_{2}^{I I}=\partial_{x}(A(U))\left(J(U) \partial_{x}^{2} \mathcal{U}, U_{x}\right), \\
G_{1}^{I}=J(U) \partial_{x}(A(U))\left(\partial_{x} \mathcal{U}, U_{x x}\right), & G_{2}^{I}=\partial_{x}(J(U)) \partial_{x}(A(U))\left(\partial_{x} \mathcal{U}, U_{x}\right), \\
G_{3}^{I}=J(U) \partial_{x}^{2}(A(U))\left(\partial_{x} \mathcal{U}, U_{x}\right), & G_{4}^{I}=J(U) \partial_{x}(A(U))\left(\mathcal{U}, U_{x x x}\right), \\
G_{5}^{I}=J(U) \partial_{x}^{3}(A(U))\left(\mathcal{U}, U_{x}\right) . &
\end{array}
$$

For $G_{1}^{I I}$, we can express by

$$
G_{1}^{I I}=T(U) \partial_{x}^{2} \mathcal{U}
$$

where $T(U)$ has been defined by (2.30).

For $G_{2}^{I I}$, we use (2.9) with $Y_{1}=J(U) \partial_{x}^{2} \mathcal{U}$ and $Y_{2}=U_{x}$ to see

$$
\begin{aligned}
G_{2}^{I I}= & \sum_{k}\left(J(U) \partial_{x}^{2} \mathcal{U}, \partial\left(D_{k}(U) P(U)\right) U_{x}\right) \nu_{k}(U) \\
& +\sum_{k}\left(\partial_{x}(P(U)) J(U) \partial_{x}^{2} \mathcal{U}, D_{k}(U) U_{x}\right) \nu_{k}(U) \\
& +\sum_{k}\left(J(U) \partial_{x}^{2} \mathcal{U}, D_{k}(U) U_{x}\right) D_{k}(U) U_{x} . \\
= & -\sum_{k}\left(\partial_{x}^{2} \mathcal{U}, J(U) D_{k}(U) U_{x}\right) D_{k}(U) U_{x}+\sum_{k} \mathcal{O}\left(\left|\partial_{x}^{2} \mathcal{U}\right|\right) \nu_{k}(U) .
\end{aligned}
$$

Moreover, from $N(U) D_{k}(U) U_{x}=\sum_{\ell}\left(D_{k}(U) U_{x}, \nu_{\ell}(U)\right) \nu_{\ell}(U)$, it follows that

$$
G_{2}^{I I}=-\sum_{k}\left(\partial_{x}^{2} \mathcal{U}, J(U) D_{k}(U) U_{x}\right) P(U) D_{k}(U) U_{x}+\sum_{k} \mathcal{O}\left(\left|\partial_{x}^{2} \mathcal{U}\right|\right) \nu_{k}(U) .
$$

Recalling $(T(U))^{*}$ has been given by (2.39), we have

$$
G_{2}^{I I}=-(T(U))^{*} \partial_{x}^{2} \mathcal{U}+\sum_{k} \mathcal{O}\left(\left|\partial_{x}^{2} \mathcal{U}\right|\right) \nu_{k}(U) .
$$

Combining (3.28) and 3.29), we have

$$
G_{1}^{I I}+G_{2}^{I I}=\left(T(U)-(T(U))^{*}\right) \partial_{x}^{2} \mathcal{U}+\sum_{k} \mathcal{O}\left(\left|\partial_{x}^{2} \mathcal{U}\right|\right) \nu_{k}(U),
$$

which combined with (2.42) shows

$$
G_{1}^{I I}+G_{2}^{I I}=-R\left(J(U) U_{x}, U_{x}\right) \partial_{x}^{2} \mathcal{U}+S_{+}(U) \partial_{x}^{2} \mathcal{U}+\sum_{k} \mathcal{O}\left(\left|\partial_{x}^{2} \mathcal{U}\right|\right) \nu_{k}(U)
$$

Here, from 3.18 , it follows that

$$
-R\left(J(U) U_{x}, U_{x}\right) \partial_{x}^{2} \mathcal{U}
$$




$$
\begin{aligned}
= & -\partial_{x}\left\{R\left(J(U) U_{x}, U_{x}\right) \partial_{x} \mathcal{U}\right\}+2 R\left(J(U) \mathcal{U}, U_{x}\right) \partial_{x} \mathcal{U} \\
& -A(U)\left(R\left(J(U) U_{x}, U_{x}\right) \partial_{x} \mathcal{U}, U_{x}\right)+\mathcal{O}\left(|U|+\left|U_{x}\right|+|\mathcal{U}|\right) .
\end{aligned}
$$

Substituting (3.32) into (3.31), and rewriting the second term of the right hand side of (3.31) in divergence form, we arrive at

$$
\begin{aligned}
G_{1}^{I I}+G_{2}^{I I}= & -\partial_{x}\left\{R\left(J(U) U_{x}, U_{x}\right) \partial_{x} \mathcal{U}\right\}+2 R\left(J(U) \mathcal{U}, U_{x}\right) \partial_{x} \mathcal{U} \\
& +\partial_{x}\left\{S_{+}(U) \partial_{x} \mathcal{U}\right\}-\partial_{x}\left(S_{+}(U)\right) \partial_{x} \mathcal{U} \\
& +\sum_{k} \mathcal{O}\left(\left|\partial_{x}^{2} \mathcal{U}\right|+\left|\partial_{x} \mathcal{U}\right|\right) \nu_{k}(U)+\mathcal{O}\left(|U|+\left|U_{x}\right|+|\mathcal{U}|\right) .
\end{aligned}
$$

For $2 G_{1}^{I}$, from 2.10 with $Y_{1}=\partial_{x} \mathcal{U}$ and $Y_{2}=U_{x}$, we see

$$
2 G_{1}^{I}=2 J(U) \sum_{k}\left(P(U) \partial_{x} \mathcal{U}, D_{k}(U) P(U) U_{x x}\right) P(U) D_{k}(U) U_{x} .
$$

Next we compute $2 G_{2}^{I}+2 G_{2}^{I}$. For $2 G_{2}^{I}$, from 2.11) with $Y_{1}=\partial_{x} \mathcal{U}$ and $Y_{2}=U_{x}$, we see

$$
\begin{aligned}
2 G_{2}^{I}= & -2 \sum_{k}\left(P(U) \partial_{x} \mathcal{U}, \partial_{x}\left(D_{k}(U) P(U)\right) U_{x}\right) J(U) D_{k}(U) U_{x} \\
& -2 \sum_{k}\left(\partial_{x}(P(U)) \partial_{x} \mathcal{U}, D_{k}(U) U_{x}\right) J(U) D_{k}(U) U_{x} \\
& -2 \sum_{k, \ell}\left(P(U) \partial_{x} \mathcal{U}, D_{k}(U) U_{x}\right)\left(D_{k}(U) U_{x}, \nu_{\ell}(U)\right) J(U) D_{\ell}(U) U_{x} \\
& +\sum_{\ell} \mathcal{O}\left(\left|\partial_{x} \mathcal{U}\right|\right) \nu_{\ell}(U) .
\end{aligned}
$$

For $2 G_{3}^{I}$, from 2.12) with $Y_{1}=\partial_{x} \mathcal{U}$ and $Y_{2}=U_{x}$, we see

$$
\begin{aligned}
2 G_{3}^{I}= & 4 \sum_{k}\left(P(U) \partial_{x} \mathcal{U}, \partial_{x}\left(D_{k}(U) P(U)\right) U_{x}\right) J(U) D_{k}(U) U_{x} \\
& +4 \sum_{k}\left(\partial_{x}(P(U)) \partial_{x} \mathcal{U}, D_{k}(U) U_{x}\right) J(U) D_{k}(U) U_{x} \\
& +2 \sum_{k}\left(P(U) \partial_{x} \mathcal{U}, D_{k}(U) U_{x}\right) J(U) \partial_{x}\left\{D_{k}(U) U_{x}\right\} .
\end{aligned}
$$

Using (2.8), we see

$$
\begin{aligned}
J(U) \partial_{x}\left\{D_{k}(U) U_{x}\right\}= & \partial_{x}\left\{J(U) D_{k}(U) U_{x}\right\}-\partial_{x}(J(U)) D_{k}(U) U_{x} \\
= & \partial_{x}\left\{J(U) D_{k}(U) U_{x}\right\}+A(U)\left(J(U) D_{k}(U) U_{x}, U_{x}\right) \\
& +\sum_{\ell}\left(D_{k}(U) U_{x}, \nu_{\ell}(U)\right) J(U) D_{\ell}(U) U_{x} .
\end{aligned}
$$

Substituting this into (3.36), and combining with (3.35), we obtain

$$
\begin{aligned}
2 G_{2}^{I}+2 G_{3}^{I}= & (4-2) \sum_{k}\left(P(U) \partial_{x} \mathcal{U}, \partial_{x}\left(D_{k}(U) P(U)\right) U_{x}\right) J(U) D_{k}(U) U_{x} \\
& +2 \sum_{k}\left(P(U) \partial_{x} \mathcal{U}, D_{k}(U) U_{x}\right) \partial_{x}\left\{J(U) D_{k}(U) U_{x}\right\}
\end{aligned}
$$




$$
\begin{aligned}
& +2 \sum_{k}\left(P(U) \partial_{x} \mathcal{U}, D_{k}(U) U_{x}\right) A(U)\left(J(U) D_{k}(U) U_{x}, U_{x}\right) \\
& +(2-2) \sum_{k, \ell}\left(P(U) \partial_{x} \mathcal{U}, D_{k}(U) U_{x}\right)\left(D_{k}(U) U_{x}, \nu_{\ell}(U)\right) J(U) D_{\ell}(U) U_{x} \\
& +(4-2) \sum_{k}\left(\partial_{x}(P(U)) \partial_{x} \mathcal{U}, D_{k}(U) U_{x}\right) J(U) D_{k}(U) U_{x} \\
& +\sum_{\ell} \mathcal{O}\left(\left|\partial_{x} \mathcal{U}\right|\right) \nu_{\ell}(U) .
\end{aligned}
$$

Since $\partial_{x}\left(D_{k}(U) P(U)\right) U_{x}=\partial_{x}\left\{D_{k}(U) U_{x}\right\}-D_{k}(U) P(U) U_{x x}$ follows from $P(U) U_{x}=U_{x}$, we obtain

$$
\begin{aligned}
2 G_{2}^{I}+2 G_{3}^{I}= & -2 \sum_{k}\left(P(U) \partial_{x} \mathcal{U}, D_{k}(U) P(U) U_{x x}\right) J(U) D_{k}(U) U_{x} \\
& +2 \sum_{k}\left(P(U) \partial_{x} \mathcal{U}, \partial_{x}\left\{D_{k}(U) U_{x}\right\}\right) J(U) D_{k}(U) U_{x} \\
& +2 \sum_{k}\left(P(U) \partial_{x} \mathcal{U}, D_{k}(U) U_{x}\right) \partial_{x}\left\{J(U) D_{k}(U) U_{x}\right\} \\
& +2 \sum_{k}\left(\partial_{x}(P(U)) \partial_{x} \mathcal{U}, D_{k}(U) U_{x}\right) J(U) D_{k}(U) U_{x} \\
& +\sum_{\ell} \mathcal{O}\left(\left|\partial_{x} \mathcal{U}\right|\right) \nu_{\ell}(U) .
\end{aligned}
$$

Furthermore, recalling $T(U)=J(U) \partial_{x}(A(U))\left(\cdot, U_{x}\right)$ by definition and using the expression (2.31) for $\partial_{x}(T(U))$, we can write

$$
\begin{aligned}
2 G_{2}^{I}+2 G_{3}^{I}= & -2 \sum_{k}\left(P(U) \partial_{x} \mathcal{U}, D_{k}(U) P(U) U_{x x}\right) J(U) D_{k}(U) U_{x} \\
& +2 \partial_{x}(T(U)) \partial_{x} \mathcal{U}+\sum_{\ell} \mathcal{O}\left(\left|\partial_{x} \mathcal{U}\right|\right) \nu_{\ell}(U) .
\end{aligned}
$$

Observing (3.34), the first term of the right hand of the above is just $-2 G_{1}^{I}$. Thus, we get

$$
2 G_{1}^{I}+2 G_{2}^{I}+2 G_{3}^{I}=2 \partial_{x}(T(U)) \partial_{x} \mathcal{U}+\sum_{\ell} \mathcal{O}\left(\left|\partial_{x} \mathcal{U}\right|\right) \nu_{\ell}(U)
$$

For the first term of the right hand side, we now apply (2.32) in Proposition 2.12 with $Y=$ $\partial_{x} \mathcal{U}$. In the computation, note first that

$$
\begin{aligned}
& J(U) R\left(U_{x}, \partial_{x}(N(U)) \partial_{x} \mathcal{U}\right) U_{x} \\
& \left.=J(U) R\left(U_{x}, P(U) \partial_{x}(N(U)) \partial_{x} \mathcal{U}\right) U_{x} \quad(\because 2.14)\right) \\
& \left.=J(U) \sum_{\ell}\left(\partial_{x} \mathcal{U}, \nu_{\ell}(U)\right) R\left(U_{x}, D_{\ell}\left(U_{x}\right) U_{x}\right) U_{x} \quad(\because 2.37)\right) \\
& =\mathcal{O}\left(|U|+\left|U_{x}\right|+|\mathcal{U}|\right)
\end{aligned}
$$

holds, where in the last equality we use $\left(\partial_{x} \mathcal{U}, \nu_{k}(U)\right)=-\left(\mathcal{U}, D_{k}(U) U_{x}\right)$ which follows from $\mathcal{U} \perp \nu_{k}(U)$. Note second that

$$
A(U)\left(J(U) R\left(U_{x}, \partial_{x} \mathcal{U}\right) U_{x}, U_{x}\right)=\sum_{\ell} \mathcal{O}\left(\left|\partial_{x} \mathcal{U}\right|\right) \nu_{\ell}(U)
$$


holds. Noting them, we apply (2.32) with $Y=\partial_{x} \mathcal{U}$, and then obtain

$$
\begin{aligned}
\partial_{x}(T(U)) \partial_{x} \mathcal{U}= & -R\left(J(U) \mathcal{U}, U_{x}\right) \partial_{x} \mathcal{U}-\frac{1}{2}\left(B_{2}(U)+B_{3}(U)\right) \partial_{x} \mathcal{U} \\
& +\sum_{k} \partial_{x}\left(\left(U_{x}, D_{k}(U) U_{x}\right) J(U) D_{k}(U) P(U)\right) \partial_{x} \mathcal{U} \\
& +\sum_{\ell} \mathcal{O}\left(\left|\partial_{x} \mathcal{U}\right|\right) \nu_{\ell}(U)+\mathcal{O}\left(|U|+\left|U_{x}\right|+|\mathcal{U}|\right) .
\end{aligned}
$$

Therefore, we obtain as follows:

$$
\begin{aligned}
2 G_{1}^{I}+2 G_{2}^{I}+2 G_{3}^{I}= & -2 R\left(J(U) \mathcal{U}, U_{x}\right) \partial_{x} \mathcal{U}-\left(B_{2}(U)+B_{3}(U)\right) \partial_{x} \mathcal{U} \\
& +2 \sum_{k} \partial_{x}\left(\left(U_{x}, D_{k}(U) U_{x}\right) J(U) D_{k}(U) P(U)\right) \partial_{x} \mathcal{U} \\
& +\sum_{\ell} \mathcal{O}\left(\left|\partial_{x} \mathcal{U}\right|\right) \nu_{\ell}(U)+\mathcal{O}\left(|U|+\left|U_{x}\right|+|\mathcal{U}|\right) .
\end{aligned}
$$

For $G_{4}^{I}$, from $(2.10)$ with $Y_{1}=\mathcal{U}=P(U) \mathcal{U}$ and $Y_{2}=U_{x x x}$, we see

$$
G_{4}^{I}=\sum_{k}\left(\mathcal{U}, D_{k}(U) P(U) U_{x x x}\right) J(U) D_{k}(U) U_{x} .
$$

Since $U_{x x x}=\partial_{x} \mathcal{U}+\mathcal{O}\left(|U|+\left|U_{x}\right|+|\mathcal{U}|\right)$, we deduce

$$
\begin{aligned}
G_{4}^{I} & =\sum_{k}\left(\mathcal{U}, D_{k}(U) P(U) \partial_{x} \mathcal{U}\right) J(U) D_{k}(U) U_{x}+\mathcal{O}\left(|U|+\left|U_{x}\right|+|\mathcal{U}|\right) \\
& =J(U) \sum_{k}\left(\mathcal{U}, D_{k}(U) P(U) \partial_{x} \mathcal{U}\right) P(U) D_{k}(U) U_{x}+\mathcal{O}\left(|U|+\left|U_{x}\right|+|\mathcal{U}|\right) .
\end{aligned}
$$

Applying (2.16) for the first term of the right hand side with $Y_{1}=U_{x}, Y_{2}=\partial_{x} \mathcal{U}, Y_{3}=\mathcal{U}$, and using $P(U) \partial_{x} \mathcal{U}=\partial_{x} \mathcal{U}+\mathcal{O}\left(|U|+\left|U_{x}\right|+|\mathcal{U}|\right)$, we deduce

$$
\begin{aligned}
G_{4}^{I}= & J(U) R\left(U_{x}, \partial_{x} \mathcal{U}\right) \mathcal{U}+J(U) \sum_{k}\left(\mathcal{U}, D_{k}(U) U_{x}\right) P(U) D_{k}(U) P(U) \partial_{x} \mathcal{U} \\
& +\mathcal{O}\left(|U|+\left|U_{x}\right|+|\mathcal{U}|\right) \\
= & -R\left(\partial_{x} \mathcal{U}, U_{x}\right) J(U) \mathcal{U}+\sum_{k}\left(\mathcal{U}, D_{k}(U) U_{x}\right) J(U) D_{k}(U) \partial_{x} \mathcal{U} \\
& +\mathcal{O}\left(|U|+\left|U_{x}\right|+|\mathcal{U}|\right) .
\end{aligned}
$$

Furthermore, applying (2.27) with $Y=\partial_{x} \mathcal{U}$, we arrive at

$$
\begin{aligned}
G_{4}^{I}= & -\frac{1}{2} R\left(J(U) \mathcal{U}, U_{x}\right) \partial_{x} \mathcal{U}+\left(\frac{1}{2} B_{1}(U)-\frac{1}{4} B_{2}(U)-\frac{1}{4} B_{3}(U)\right) \partial_{x} \mathcal{U} \\
& +\sum_{k}\left(\mathcal{U}, D_{k}(U) U_{x}\right) J(U) D_{k}(U) \partial_{x} \mathcal{U}+\mathcal{O}\left(|U|+\left|U_{x}\right|+|\mathcal{U}|\right) .
\end{aligned}
$$

For $G_{5}^{I}=J(U) \partial_{x}^{3}(A(U))\left(\mathcal{U}, U_{x}\right)$, starting from taking the partial derivative of (2.9) twice with respect to $x$, we see

$$
\begin{aligned}
& \partial_{x}^{3}(A(U))\left(\mathcal{U}, U_{x}\right) \\
& =\sum_{k}\left(P(U) \mathcal{U}, \partial_{x}^{3}\left(D_{k}(U) P(U)\right) U_{x}\right) \nu_{k}(U)+\sum_{k}\left(\partial_{x}^{3}(P(U)) \mathcal{U}, D_{k}(U) P(U) U_{x}\right) \nu_{k}(U)
\end{aligned}
$$




$$
+\sum_{k}\left(P(U) \mathcal{U}, D_{k}(U) P(U) U_{x}\right) D_{k}(U) U_{x x x}+\mathcal{O}\left(|U|+\left|U_{x}\right|+\left|U_{x x}\right|\right) .
$$

Since $J(U) \nu_{k}(U)=0$ and $U_{x x x}=\partial_{x} \mathcal{U}+\mathcal{O}\left(|U|+\left|U_{x}\right|+|\mathcal{U}|\right)$, we have

$$
G_{5}^{I}=\sum_{k}\left(\mathcal{U}, D_{k}(U) U_{x}\right) J(U) D_{k}(U) \partial_{x} \mathcal{U}+\mathcal{O}\left(|U|+\left|U_{x}\right|+|\mathcal{U}|\right)
$$

Gathering the information (3.33), 3.38), 3.40) and (3.41), we obtain

$$
\begin{aligned}
G_{1}^{I I} & +G_{2}^{I I}+2 G_{1}^{I}+2 G_{2}^{I}+2 G_{3}^{I}+G_{4}^{I}+G_{5}^{I} \\
= & -\partial_{x}\left\{R\left(J(U) U_{x}, U_{x}\right) \partial_{x} \mathcal{U}\right\}+\partial_{x}\left\{S_{+}(U) \partial_{x} \mathcal{U}\right\} \\
& +\partial_{x}\left(-S_{+}(U)+2 \sum_{k}\left(U_{x}, D_{k}(U) U_{x}\right) P(U) J(U) D_{k}(U) P(U)\right) \partial_{x} \mathcal{U} \\
& -\frac{1}{2} R\left(J(U) \mathcal{U}, U_{x}\right) \partial_{x} \mathcal{U}+\left(\frac{1}{2} B_{1}(U)-\frac{5}{4} B_{2}(U)-\frac{5}{4} B_{3}(U)\right) \partial_{x} \mathcal{U} \\
& +2 \sum_{k}\left(\mathcal{U}, D_{k}(U) U_{x}\right) J(U) D_{k}(U) \partial_{x} \mathcal{U} \\
& +\sum_{k} \mathcal{O}\left(\left|\partial_{x}^{2} \mathcal{U}\right|+\left|\partial_{x} \mathcal{U}\right|\right) \nu_{k}(U)+\mathcal{O}\left(|U|+\left|U_{x}\right|+|\mathcal{U}|\right) \\
= & -\partial_{x}\left\{R\left(J(U) U_{x}, U_{x}\right) \partial_{x} \mathcal{U}\right\}+\partial_{x}\left\{S_{+}(U) \partial_{x} \mathcal{U}\right\}+\partial_{x}\left(S_{-}(U)\right) \partial_{x} \mathcal{U} \\
& -\frac{1}{2} R\left(J(U) \mathcal{U}, U_{x}\right) \partial_{x} \mathcal{U}+\left(\frac{1}{2} B_{1}(U)-\frac{5}{4} B_{2}(U)-\frac{5}{4} B_{3}(U)\right) \partial_{x} \mathcal{U} \\
& +2 \sum_{k}\left(\mathcal{U}, D_{k}(U) U_{x}\right) J(U) D_{k}(U) \partial_{x} \mathcal{U} \\
& +\sum_{k} \mathcal{O}\left(\left|\partial_{x}^{2} \mathcal{U}\right|+\left|\partial_{x} \mathcal{U}\right|\right) \nu_{k}(U)+\mathcal{O}\left(|U|+\left|U_{x}\right|+|\mathcal{U}|\right) .
\end{aligned}
$$

Substituting (3.42) into 3.27), we get the expression of $I(U)=d w\left(\nabla_{x}^{2} J_{u} \nabla_{x}^{3} u_{x}\right)$. Consequently, substituting it multiplied by $a$ into (3.23), and combining with (3.24), we derive the desired (3.1) with (3.2).

3.2. Classical Energy Estimate for $\mathcal{W}$ in $L^{2}$. We derive the classical energy estimate for $\mathcal{W}$ in $L^{2}$. In what follows, the inner product and the norm in $L^{2}$ for $\mathbb{R}^{d}$-valued functions on $\mathbb{T}$ will be denoted by $\langle\cdot\rangle$ and $\|\cdot\|_{L^{2}}$ respectively. That is, for $\phi, \psi: \mathbb{T} \rightarrow \mathbb{R}^{d},\langle\phi, \psi\rangle$ and $\|\phi\|_{L^{2}}$ is given by $\langle\phi, \psi\rangle=\int_{\mathbb{T}}(\phi(x), \psi(x)) d x$ and $\|\phi\|_{L^{2}}=\sqrt{\langle\phi, \phi\rangle}$ respectively. In the same way, the standard Sobolev norm in $H^{k}\left(\mathbb{T} ; \mathbb{R}^{d}\right)$ with integer $k \geqslant 1$ is denoted by $\|\cdot\|_{H^{k}}$. Moreover, we recall the following properties hold:

$$
\begin{aligned}
& \mathcal{U}, \mathcal{V}, \mathcal{W} \in L^{\infty}\left(-T, T ; H^{4}\left(\mathbb{T} ; \mathbb{R}^{d}\right)\right) \cap C\left([-T, T] ; H^{4}\left(\mathbb{T} ; \mathbb{R}^{d}\right)\right), \\
& \partial_{x}^{\ell} U_{x}, \partial_{x}^{\ell} V_{x} \in L^{\infty}\left(-T, T ; C\left(\mathbb{T} ; \mathbb{R}^{d}\right)\right) \quad(\ell=0,1, \ldots, 4), \\
& \partial_{x}^{\ell} \mathcal{U}, \partial_{x}^{\ell} \mathcal{V} \in L^{\infty}\left(-T, T ; C\left(\mathbb{T} ; \mathbb{R}^{d}\right)\right) \quad(\ell=0,1, \ldots, 3),
\end{aligned}
$$

since $k \geqslant 5$ is imposed.

Noting $\mathcal{V}$ also satisfies (3.1) replacing $\left(U, U_{x}, \mathcal{U}\right)$ with $\left(V, V_{x}, \mathcal{V}\right)$, we take the difference of $\partial_{t} \mathcal{U}$ and $\partial_{t} \mathcal{V}$, which yields

$$
\partial_{t} \mathcal{W}=L(U) \mathcal{W}+a(J(U)-J(V)) \partial_{x}^{4} \mathcal{V}
$$




$$
+\sum_{k} \mathcal{O}\left(\left|\partial_{x}^{2} \mathcal{W}\right|+\left|\partial_{x} \mathcal{W}\right|\right) \nu_{k}(U)+\mathcal{O}\left(|Z|+\left|Z_{x}\right|+|\mathcal{W}|\right)
$$

for almost every $t \in(-T, T)$, where $L(U)$ is the partial differential operator given in Proposition 3.1. Since $\mathcal{W} \in L^{\infty}\left(-T, T ; H^{4}\left(\mathbb{T} ; \mathbb{R}^{d}\right)\right)$ as commented above, we see $\partial_{t} \mathcal{W} \in$ $L^{\infty}\left(-T, T ; L^{2}\left(\mathbb{T} ; \mathbb{R}^{d}\right)\right)$ holds. Hence, following the argument in, e.g., [42, Lemma 3.2] or [31, Theorem 7.2], we can show

$$
\frac{1}{2} \frac{d}{d t}\|\mathcal{W}(t)\|_{L^{2}}^{2}=\left\langle\partial_{t} \mathcal{W}(t), \mathcal{W}(t)\right\rangle
$$

holds for almost every $t \in(-T, T)$. Using (3.43) and (3.2), we have

$$
\begin{aligned}
\left\langle\partial_{t}\right. & \mathcal{W}, \mathcal{W}\rangle \\
= & a\left\langle\partial_{x}^{2}\left\{J(U) \partial_{x}^{2} \mathcal{W}\right\}, \mathcal{W}\right\rangle+2 a\left\langle A(U)\left(J(U) \partial_{x}^{3} \mathcal{W}, U_{x}\right), \mathcal{W}\right\rangle \\
& +\lambda\left\langle\partial_{x}\left\{J(U) \partial_{x} \mathcal{W}\right\}, \mathcal{W}\right\rangle+(-a+b)\left\langle R\left(\partial_{x}^{2} \mathcal{W}, J(U) U_{x}\right) U_{x}, \mathcal{W}\right\rangle \\
& +(-a+b+c)\left\langle\partial_{x}\left\{R\left(J(U) U_{x}, U_{x}\right) \partial_{x} \mathcal{W}\right\}, \mathcal{W}\right\rangle \\
& +\left(-\frac{a}{2}-\frac{b}{2}+3 c\right)\left\langle R\left(J(U) \mathcal{U}, U_{x}\right) \partial_{x} \mathcal{W}, \mathcal{W}\right\rangle+\sum_{i=1}^{3} c_{i}\left\langle B_{i}(U) \partial_{x} \mathcal{W}, \mathcal{W}\right\rangle \\
& +a\left\langle\partial_{x}\left\{S_{+}(U) \partial_{x} \mathcal{W}\right\}, \mathcal{W}\right\rangle+a\left\langle\partial_{x}\left(S_{-}(U)\right) \partial_{x} \mathcal{W}, \mathcal{W}\right\rangle \\
& +2 a\left\langle\sum_{k}\left(\mathcal{U}, D_{k}(U) U_{x}\right) J(U) D_{k}(U) \partial_{x} \mathcal{W}, \mathcal{W}\right\rangle \\
& +a\left\langle(J(U)-J(V)) \partial_{x}^{4} \mathcal{V}, \mathcal{W}\right\rangle \\
& +\left\langle\sum_{k} \mathcal{O}\left(\left|\partial_{x}^{2} \mathcal{W}\right|+\left|\partial_{x} \mathcal{W}\right|\right) \nu_{k}(U), \mathcal{W}\right\rangle+\left\langle\mathcal{O}\left(|Z|+\left|Z_{x}\right|+|\mathcal{W}|\right), \mathcal{W}\right\rangle
\end{aligned}
$$

for almost every $t \in(-T, T)$. We furthermore compute the right hand side of the above term by term. Before that, we observe some key properties related to $\nu_{k}(U)$ and $D_{k}(U)$ for $k=2 n+1 \ldots, d$.

Proposition 3.2. Under the same setting as above, the following properties hold.

$$
\begin{aligned}
\left(\nu_{k}(U), \mathcal{W}\right) & =-\left(\nu_{k}(U)-\nu_{k}(V), \mathcal{V}\right)=\mathcal{O}(|Z|), \\
\left(\nu_{k}(U), \partial_{x} \mathcal{W}\right) & =-\left(D_{k}(U) U_{x}, \mathcal{W}\right)-\left(D_{k}(U) Z_{x}, \mathcal{V}\right)+\mathcal{O}(|Z|) .
\end{aligned}
$$

The proof is given in [29, Lemma 3.1], and thus we omit the detail. In view of (3.46), we find that the terms including $\partial_{x}^{2} \mathcal{W}$ or $\partial_{x} \mathcal{W}$ can be handled as a harmless term if they are expressed as a linear combination of $\nu_{2 n+1}(U), \ldots, \nu_{d}(U)$. See, e.g., the argument to show (3.51). Here and hereafter, various positive constants depending on $\left\|U_{x}\right\|_{L^{\infty}\left(-T, T ; H^{5}\right)}$ and $\left\|V_{x}\right\|_{L^{\infty}\left(-T, T ; H^{5}\right)}$ will be denoted by the same $C$ without any comments. Besides, we define $D(t)$ so that the square is given by

$$
D(t)^{2}=\|Z(t)\|_{L^{2}}^{2}+\left\|Z_{x}(t)\right\|_{L^{2}}^{2}+\|\mathcal{W}(t)\|_{L^{2}}^{2} .
$$

Going back to (3.45), we use the integration by parts and the skew-symmetry of $J(U)$ to see

$$
\begin{aligned}
& a\left\langle\partial_{x}^{2}\left\{J(U) \partial_{x}^{2} \mathcal{W}\right\}, \mathcal{W}\right\rangle=a\left\langle J(U) \partial_{x}^{2} \mathcal{W}, \partial_{x}^{2} \mathcal{W}\right\rangle=0 \\
& \lambda\left\langle\partial_{x}\left\{J(U) \partial_{x} \mathcal{W}\right\}, \mathcal{W}\right\rangle=-\lambda\left\langle J(U) \partial_{x} \mathcal{W}, \partial_{x} \mathcal{W}\right\rangle=0
\end{aligned}
$$


By the Cauchy-Schwartz inequality, we deduce

$$
\left\langle\mathcal{O}\left(|Z|+\left|Z_{x}\right|+|\mathcal{W}|\right), \mathcal{W}\right\rangle \leqslant\left\|\mathcal{O}\left(|Z|+\left|Z_{x}\right|+|\mathcal{W}|\right)\right\|_{L^{2}}\|\mathcal{W}\|_{L^{2}} \leqslant C D(t)^{2} .
$$

By (3.46), we see $\left(\nu_{k}(U), \mathcal{W}\right)=\mathcal{O}(|Z|)$. Thus, integrating by parts, we obtain

$$
\left\langle\sum_{k} \mathcal{O}\left(\left|\partial_{x}^{2} \mathcal{W}\right|+\left|\partial_{x} \mathcal{W}\right|\right) \nu_{k}(U), \mathcal{W}\right\rangle \leqslant C D(t)^{2}
$$

Integrating by parts, and then using 2.18)-2.19), we have

$$
\left\langle\partial_{x}\left\{R\left(J(U) U_{x}, U_{x}\right) \partial_{x} \mathcal{W}\right\}, \mathcal{W}\right\rangle=-\left\langle R\left(J(U) U_{x}, U_{x}\right) \partial_{x} \mathcal{W}, \partial_{x} \mathcal{W}\right\rangle=0 .
$$

Noting the symmetry of $B_{i}(U)$ discussed in Proposition 2.10, and integrating by parts, we deduce

$$
\left\langle B_{i}(U) \partial_{x} \mathcal{W}, \mathcal{W}\right\rangle=-\frac{1}{2}\left\langle\partial_{x}\left(B_{i}(U)\right) \mathcal{W}, \mathcal{W}\right\rangle \leqslant C D(t)^{2} .
$$

Recall that $S_{+}(U)$ and $S_{-}(U)$ defined in Definition 2.14 or (2.42)-(2.43) are respectively skew-symmetric and symmetric, that is, $\left(S_{ \pm}(U) Y_{1}, Y_{2}\right)=\mp\left(Y_{1}, S_{ \pm}(U) Y_{2}\right)$ for any $Y_{1}, Y_{2}$ : $[-T, T] \times \mathbb{T} \rightarrow \mathbb{R}^{d}$. Using the skew-symmetry of $S_{+}(U)$ and the symmetry of $\partial_{x}\left(S_{-}(U)\right)$, we have

$$
\begin{aligned}
& \left\langle\partial_{x}\left\{S_{+}(U) \partial_{x} \mathcal{W}\right\}, \mathcal{W}\right\rangle=-\left\langle S_{+}(U) \partial_{x} \mathcal{W}, \partial_{x} \mathcal{W}\right\rangle=0, \\
& \left\langle\partial_{x}\left(S_{-}(U)\right) \partial_{x} \mathcal{W}, \mathcal{W}\right\rangle \leqslant C D(t)^{2}
\end{aligned}
$$

By the Cauchy-Schwartz inequality, the Sobolev embedding $H^{1}\left(\mathbb{T} ; \mathbb{R}^{d}\right) \subset C\left(\mathbb{T} ; \mathbb{R}^{d}\right)$, and $\mathcal{V} \in L^{\infty}\left(-T, T ; H^{4}\left(\mathbb{T} ; \mathbb{R}^{d}\right)\right)$, we deduce

$$
\begin{aligned}
\left\langle\left(J(U)-J(V) \partial_{x}^{4} \mathcal{V}, \mathcal{W}\right\rangle\right. & \leqslant\left\|(J(U)-J(V)) \partial_{x}^{4} \mathcal{V}\right\|_{L^{2}}\|\mathcal{W}\|_{L^{2}} \\
& \leqslant C\|Z\|_{H^{1}}\left\|\partial_{x}^{4} \mathcal{V}\right\|_{L^{2}}\|\mathcal{W}\|_{L^{2}} \\
& \leqslant C D(t)^{2} .
\end{aligned}
$$

We next set $E:=\left\langle A(U)\left(J(U) \partial_{x}^{3} \mathcal{W}, U_{x}\right), \mathcal{W}\right\rangle$. Although the third order term $\partial_{x}^{3} \mathcal{W}$ is seemingly included in $E$, we will see that only a first-order loss of derivative occurs thanks to the property of $A(U)(\cdot, \cdot)$ as demonstrated below. Noting $A(U)\left(\mathcal{O}\left(\left|\partial_{x}^{2} \mathcal{W}\right|\right),\left|U_{x}\right|+\left|U_{x x}\right|\right)=$ $\sum_{k} \mathcal{O}\left(\left|\partial_{x}^{2} \mathcal{W}\right|\right) \nu_{k}(U)$ and (3.46), we use the integration by parts to obtain

$$
\begin{aligned}
2 E^{I I I} \leqslant & -2\left\langle\partial_{x}(A(U))\left(J(U) \partial_{x}^{2} \mathcal{W}, U_{x}\right), \mathcal{W}\right\rangle \\
& -2\left\langle A(U)\left(J(U) \partial_{x}^{2} \mathcal{W}, \partial_{x} \mathcal{W}\right)\right\rangle+C D(t)^{2}
\end{aligned}
$$

By (2.9) and (3.46), we see

$$
\begin{aligned}
& \left(\partial_{x}(A(U))\left(J(U) \partial_{x}^{2} \mathcal{W}, U_{x}\right), \mathcal{W}\right) \\
& =\sum_{k}\left(J(U) \partial_{x}^{2} \mathcal{W}, \partial_{x}\left(D_{k}(U) P(U)\right) U_{x}\right)\left(\nu_{k}(U), \mathcal{W}\right) \\
& \quad+\sum_{k}\left(J(U) \partial_{x}^{2} \mathcal{W}, D_{k}(U) U_{x}\right)\left(D_{k}(U) U_{x}, \mathcal{W}\right) \\
& \quad+\sum_{k}\left(\partial_{x}(P(U)) J(U) \partial_{x}^{2} \mathcal{W}, D_{k}(U) U_{x}\right)\left(\nu_{k}(U), \mathcal{W}\right) \\
& =\sum_{k}\left(J(U) \partial_{x}^{2} \mathcal{W}, D_{k}(U) U_{x}\right)\left(D_{k}(U) U_{x}, \mathcal{W}\right)
\end{aligned}
$$




$$
\begin{aligned}
& +\sum_{k}\left(J(U) \partial_{x}^{2} \mathcal{W}, \partial_{x}\left(D_{k}(U) P(U)\right) U_{x}\right) \mathcal{O}(|Z|) \\
& +\sum_{k}\left(\partial_{x}\left(P(U) J(U) \partial_{x}^{2} \mathcal{W}, D_{k}(U) U_{x}\right) \mathcal{O}(|Z|)\right.
\end{aligned}
$$

By (3.47), we see

$$
\begin{aligned}
\left(A(U)\left(J(U) \partial_{x}^{2} \mathcal{W}, U_{x}\right), \partial_{x} \mathcal{W}\right)= & \sum_{k}\left(J(U) \partial_{x}^{2} \mathcal{W}, D_{k}(U) U_{x}\right)\left(\nu_{k}(U), \partial_{x} \mathcal{W}\right) \\
= & -\sum_{k}\left(J(U) \partial_{x}^{2} \mathcal{W}, D_{k}(U) U_{x}\right)\left(D_{k}(U) U_{x}, \mathcal{W}\right) \\
& -\sum_{k}\left(J(U) \partial_{x}^{2} \mathcal{W}, D_{k}(U) U_{x}\right)\left(D_{k}(U) Z_{x}, \mathcal{V}\right) \\
& -\sum_{k}\left(J(U) \partial_{x}^{2} \mathcal{W}, D_{k}(U) U_{x}\right) \mathcal{O}(|Z|)
\end{aligned}
$$

Combining (3.58) and (3.59), and using the integration by parts, we have

$$
\begin{aligned}
2 E & \leqslant 2\left\langle\sum_{k}\left(J(U) \partial_{x}^{2} \mathcal{W}, D_{k}(U) U_{x}\right) D_{k}(U) Z_{x}, \mathcal{V}\right\rangle+C D(t)^{2} \\
& \leqslant 2\left\langle\sum_{k}\left(J(U) \mathcal{W}, D_{k}(U) U_{x}\right) D_{k}(U) Z_{x x x}, \mathcal{V}\right\rangle+C D(t)^{2} .
\end{aligned}
$$

Furthermore, since $\left(D_{k}(U) Z_{x x x}, \mathcal{V}\right)=\left(D_{k}(U) Z_{x x x},-\mathcal{W}+\mathcal{U}\right)=\left(D_{k}(U) Z_{x x x}, \mathcal{U}\right)+\mathcal{O}(|\mathcal{W}|)$ and $Z_{x x x}=P(U) \partial_{x} \mathcal{W}+\mathcal{O}\left(|Z|+\left|Z_{x}\right|+|\mathcal{W}|\right)$, we see

$$
\left(D_{k}(U) Z_{x x x}, \mathcal{V}\right)=\left(D_{k}(U) P(U) \partial_{x} \mathcal{W}, \mathcal{U}\right)+\mathcal{O}\left(|Z|+\left|Z_{x}\right|+|\mathcal{W}|\right)
$$

Substituting this into 3.60, we obtain

$$
2 E \leqslant 2\left\langle\sum_{k}\left(J(U) \mathcal{W}, D_{k}(U) U_{x}\right) D_{k}(U) P(U) \partial_{x} \mathcal{W}, \mathcal{U}\right\rangle+C D(t)^{2} .
$$

Furthermore, using $N(U) D_{k}(U) P(U) \partial_{x} \mathcal{W} \perp \mathcal{U}$, we have

$$
2 E \leqslant 2\left\langle\sum_{k}\left(J(U) \mathcal{W}, D_{k}(U) U_{x}\right) P(U) D_{k}(U) P(U) \partial_{x} \mathcal{W}, \mathcal{U}\right\rangle+C D(t)^{2} .
$$

Then, applying (2.16) with $Y_{1}=\partial_{x} \mathcal{W}, Y_{2}=U_{x} Y_{3}=J(U) \mathcal{W}$, we obtain

$$
\begin{aligned}
2 E \leqslant & 2\left\langle R\left(\partial_{x} \mathcal{W}, U_{x}\right) J(U) \mathcal{W}, \mathcal{U}\right\rangle \\
& +2\left\langle\sum_{k}\left(J(U) \mathcal{W}, D_{k}(U) P(U) \partial_{x} \mathcal{W}\right) P(U) D_{k}(U) U_{x}, \mathcal{U}\right\rangle+C D(t)^{2} .
\end{aligned}
$$

Using (2.19) and (2.23) for the first term, and $N(U) \partial_{x} \mathcal{W}=\mathcal{O}\left(|Z|+\left|Z_{x}\right|+|\mathcal{W}|\right)$ and $\left(P(U) D_{k}(U) U_{x}, \mathcal{U}\right)=\left(D_{k}(U) U_{x}, P(U) \mathcal{U}\right)=\left(D_{k}(U) U_{x}, \mathcal{U}\right)$ for the second term, we deduce

$$
2 E \leqslant 2\left\langle R\left(\partial_{x} \mathcal{W}, U_{x}\right) J(U) \mathcal{U}, \mathcal{W}\right\rangle
$$




$$
\begin{aligned}
& +2\left\langle\sum_{k}\left(P(U) D_{k}(U) U_{x}, \mathcal{U}\right) D_{k}(U) P(U) \partial_{x} \mathcal{W}, J(U) \mathcal{W}\right\rangle+C D(t)^{2} \\
\leqslant & 2\left\langle R\left(\partial_{x} \mathcal{W}, U_{x}\right) J(U) \mathcal{U}, \mathcal{W}\right\rangle \\
& -2\left\langle\sum_{k}\left(\mathcal{U}, D_{k}(U) U_{x}\right) J(U) D_{k}(U) \partial_{x} \mathcal{W}, \mathcal{W}\right\rangle+C D(t)^{2} .
\end{aligned}
$$

Applying (2.27) in Proposition 2.11 with $Y=\partial_{x} \mathcal{W}$, and noting $\left\langle B_{i}(U) \partial_{x} \mathcal{W}, \mathcal{W}\right\rangle \leqslant C D(t)^{2}$ follows from the symmetry of $\overline{B_{i}(U)}$ as we obtain (3.53), we obtain

$$
\begin{aligned}
2 a & \left\langle A(U)\left(J(U) \partial_{x}^{3} \mathcal{W}, U_{x}\right), \mathcal{W}\right\rangle(=2 a E) \\
\leqslant & a\left\langle R\left(J(U) \mathcal{U}, U_{x}\right) \partial_{x} \mathcal{W}, \mathcal{W}\right\rangle \\
& -2 a\left\langle\sum_{k}\left(\mathcal{U}, D_{k}(U) U_{x}\right) J(U) D_{k}(U) \partial_{x} \mathcal{W}, \mathcal{W}\right\rangle+C D(t)^{2} .
\end{aligned}
$$

Finally, by substituting (3.48)-(3.56), and 3.63) into 3.45) combined with (3.44), we conclude

$$
\begin{aligned}
\frac{1}{2} \frac{d}{d t}\|\mathcal{W}\|_{L^{2}}^{2} \leqslant & (-a+b)\left\langle R\left(\partial_{x}^{2} \mathcal{W}, J(U) U_{x}\right) U_{x}, \mathcal{W}\right\rangle \\
& +\left(a-\frac{a}{2}-\frac{b}{2}+3 c\right)\left\langle R\left(J(U) \mathcal{U}, U_{x}\right) \partial_{x} \mathcal{W}, \mathcal{W}\right\rangle \\
& +(2 a-2 a)\left\langle\sum_{k}\left(\mathcal{U}, D_{k}(U) U_{x}\right) J(U) D_{k}(U) \partial_{x} \mathcal{W}, \mathcal{W}\right\rangle+C D(t)^{2} \\
= & (-a+b)\left\langle R\left(\partial_{x}^{2} \mathcal{W}, J(U) U_{x}\right) U_{x}, \mathcal{W}\right\rangle \\
& +\left(\frac{a}{2}-\frac{b}{2}+3 c\right)\left\langle R\left(J(U) \mathcal{U}, U_{x}\right) \partial_{x} \mathcal{W}, \mathcal{W}\right\rangle+C D(t)^{2}
\end{aligned}
$$

holds for almost every $t \in(-T, T)$. This combined with the integration by parts implies only the estimate of the form $\frac{1}{2} \frac{d}{d t}\|\mathcal{W}\|_{L^{2}}^{2} \leqslant C\|\mathcal{W}\|_{H^{1}}^{2}$, which is still unsatisfactory.

\section{Proof of Theorem 1.3}

In this section, we complete the proof of Theorem 1.3 .

Proof of Theorem 1.3. Suppose $u$ and $v$ are solutions to (1.4)-(1.5) in Theorem 1.2. We shall show $u=v$. To do this, we fix $w$ as an isometric embedding of $(N, J, h)$ into an Euclidean space $\mathbb{R}^{d}$, and set $U=w \circ u, V=w \circ v, Z=U-V, \mathcal{U}=d w_{u}\left(\nabla_{x} u_{x}\right), \mathcal{V}=d w_{v}\left(\nabla_{x} v_{x}\right)$, and $\mathcal{W}=\mathcal{U}-\mathcal{V}$. The setting is completely the same as that in the previous section. Hence, we do not repeat but it is recommendable to recall the comments stated at the beginning of Section 3 .

For this purpose, as $w$ is injective, it suffices to show $Z=0$. We consider the estimate for the following modified energy

$$
\begin{aligned}
\widetilde{D}(t)^{2} & :=\|Z(t)\|_{L^{2}}^{2}+\left\|Z_{x}(t)\right\|_{L^{2}}^{2}+\|\widetilde{\mathcal{W}}(t)\|_{L^{2}}^{2} \\
\widetilde{\mathcal{W}} & :=\mathcal{W}+\Lambda
\end{aligned}
$$


where

$$
\Lambda:=-\frac{e_{1}}{2 a} R\left(Z, U_{x}\right) U_{x}+\frac{e_{2}}{8 a} R\left(J(U) U_{x}, U_{x}\right) J(U) Z,
$$

and $e_{1}, e_{2} \in \mathbb{R}$ are constants which will be taken later. Since $u$ and $v$ satisfy the same initial value, $\widetilde{D}(0)=0$ holds. We shall show that there exists a positive constant $C$ such that

$$
\frac{1}{2} \frac{d}{d t} \widetilde{D}(t)^{2} \leqslant C \widetilde{D}(t)^{2}
$$

for almost every $t \in(-T, T)$. If it actually holds, then (4.4) together with $\widetilde{D}(0)=0$ shows

$$
\widetilde{D}(t)^{2}=\widetilde{D}(0)^{2}+\int_{0}^{t} \frac{d}{d t} \widetilde{D}(s)^{2} d s \leqslant 2 C \int_{0}^{t} \widetilde{D}(s)^{2} d s
$$

for all $t \in(-T, T)$. Since $\widetilde{D}^{2}(t)$ is a continuous real valued function on $[-T, T]$, the inequality shows $\widetilde{D}(t) \equiv 0$, which yields $Z(t)=0$ for all $t \in[-T, T]$.

In the same way as we obtain (3.64), it is now not difficult to obtain the following estimate for almost every $t \in(-T, T)$, permitting the loss of derivatives of order one:

$$
\frac{1}{2} \frac{d}{d t}\left\{\|Z(t)\|_{L^{2}}^{2}+\left\|Z_{x}(t)\right\|_{L^{2}}^{2}\right\} \leqslant C \widetilde{D}(t)^{2} .
$$

Hence, we hereafter concentrate on deriving the equation satisfied by $\widetilde{\mathcal{W}}$ and the estimate for $\|\widetilde{\mathcal{W}}(t)\|_{L^{2}}^{2}$. Observing $\Lambda=\mathcal{O}(|Z|)$, we see $\widetilde{\mathcal{W}}=\mathcal{W}+\mathcal{O}(|Z|), \partial_{x} \widetilde{\mathcal{W}}=\partial_{x} \mathcal{W}+\mathcal{O}\left(|Z|+\left|Z_{x}\right|\right)$, and $\partial_{x}^{2} \widehat{\mathcal{W}}=\partial_{x}^{2} \mathcal{W}+\mathcal{O}\left(|Z|+\left|Z_{x}\right|+|\mathcal{W}|\right)$, which will be often used without comments. Particularly, we will sometimes write $f \equiv g$ for $f, g:(-T, T) \times \mathbb{T} \rightarrow \mathbb{R}^{d}$ if $f-g=$ $\mathcal{O}\left(|Z|+\left|Z_{x}\right|+|\mathcal{W}|\right)$ holds almost everywhere. Moreover, we will sometimes use the property (2.14) without comments.

We start the computation of $\partial_{t} \widetilde{\mathcal{W}}=\partial_{t} \mathcal{W}+\partial_{t} \Lambda$. Using (2.24) and noting $\partial_{t} U_{x}=\partial_{x} U_{t} \in$ $L^{\infty}\left(-T ; T ; C\left(\mathbb{T} ; \mathbb{R}^{d}\right)\right)$, we see

$$
\begin{aligned}
\partial_{t} \Lambda & =-\frac{e_{1}}{2 a} R\left(\partial_{t}\{P(U) Z\}, U_{x}\right) U_{x}+\frac{e_{2}}{8 a} R\left(J(U) U_{x}, U_{x}\right) J(U) \partial_{t}\{P(U) Z\}+\mathcal{O}(|Z|) \\
& =-\frac{e_{1}}{2 a} R\left(P(U) Z_{t}, U_{x}\right) U_{x}+\frac{e_{2}}{8 a} R\left(J(U) U_{x}, U_{x}\right) J(U) P(U) Z_{t}+\mathcal{O}(|Z|)
\end{aligned}
$$

holds for almost every $t$. Recalling (3.7) and using (3.47) and (2.8), we see

$$
\begin{aligned}
Z_{t} & \equiv a \partial_{x}\left\{J(U) \partial_{x} \mathcal{W}\right\}+a A(U)\left(J(U) \partial_{x} \mathcal{W}, U_{x}\right) \\
& =a J(U) \partial_{x}^{2} \mathcal{W}+a \partial_{x}(J(U)) \partial_{x} \mathcal{W}+a A(U)\left(J(U) \partial_{x} \mathcal{W}, U_{x}\right) \\
& \equiv a J(U) \partial_{x}^{2} \mathcal{W} .
\end{aligned}
$$

Moreover, since

$$
\begin{aligned}
\mathcal{W} & =U_{x x}+A(U)\left(U_{x}, U_{x}\right)-V_{x x}-A(V)\left(V_{x}, V_{x}\right) \\
& =Z_{x x}+A(U)\left(U_{x}, Z_{x}\right)+A(U)\left(Z_{x}, U_{x}\right)+(A(U)-A(V))\left(V_{x}, V_{x}\right)
\end{aligned}
$$

and $J(U) A(U)(\cdot, \cdot)=0$, we see

$$
J(U) \partial_{x}^{2} \mathcal{W} \equiv J(U)\left(\partial_{x}^{2} Z_{x x}+A(U)\left(U_{x}, \partial_{x} Z_{x x}\right)+A(U)\left(\partial_{x} Z_{x x}, U_{x}\right)\right)=J(U) \partial_{x}^{2} Z_{x x}
$$

This implies

$$
Z_{t} \equiv a J(U) \partial_{x}^{2} Z_{x x}
$$


From this and (4.6), we have

$$
\partial_{t} \Lambda \equiv-\frac{e_{1}}{2} R\left(J(U) \partial_{x}^{2} Z_{x x}, U_{x}\right) U_{x}-\frac{e_{2}}{8} R\left(J(U) U_{x}, U_{x}\right) \partial_{x}^{2} Z_{x x}
$$

On the other hand, recalling (3.43), we can write

$$
\begin{aligned}
\partial_{t} \mathcal{W}= & L(U) \mathcal{W}+a(J(U)-J(V)) \partial_{x}^{4} \mathcal{V} \\
& +\sum_{k} \mathcal{O}\left(\left|\partial_{x}^{2} \mathcal{W}\right|+\left|\partial_{x} \mathcal{W}\right|\right) \nu_{k}(U)+\mathcal{O}\left(|Z|+\left|Z_{x}\right|+|\mathcal{W}|\right) \\
\equiv & L(U) \widetilde{\mathcal{W}}-L(U) \Lambda+a(J(U)-J(V)) \partial_{x}^{4} \mathcal{V} \\
& +\sum_{k} \mathcal{O}\left(\left|\partial_{x}^{2} \mathcal{W}\right|+\left|\partial_{x} \mathcal{W}\right|\right) \nu_{k}(U)
\end{aligned}
$$

Here, noting $\Lambda=\mathcal{O}(|Z|)$, it is immediate to see

$$
L(U) \Lambda \equiv a \partial_{x}^{2}\left\{J(U) \partial_{x}^{2} \Lambda\right\}+2 a A(U)\left(J(U) \partial_{x}^{3} \Lambda, U_{x}\right) .
$$

The second term of the right hand side is harmless in that

$$
A(U)\left(J(U) \partial_{x}^{3} \Lambda, U_{x}\right)=\sum_{k} \mathcal{O}\left(\left|\partial_{x}^{3} \Lambda\right|\right) \nu_{k}(U)=\sum_{k} \mathcal{O}\left(\left|\partial_{x} \mathcal{W}\right|\right) \nu_{k}(U) .
$$

Hence we obtain

$$
\begin{aligned}
L(U) \Lambda \equiv & a \partial_{x}^{2}\left\{J(U) \partial_{x}^{2} \Lambda\right\}+\sum_{k} \mathcal{O}\left(\left|\partial_{x} \mathcal{W}\right|\right) \nu_{k}(U) \\
= & -\frac{e_{1}}{2} \partial_{x}^{2}\left\{J(U) \partial_{x}^{2}\left\{R\left(Z, U_{x}\right) U_{x}\right\}\right\} \\
& +\frac{e_{2}}{8} \partial_{x}^{2}\left\{J(U) \partial_{x}^{2}\left\{R\left(J(U) U_{x}, U_{x}\right) J(U) Z\right\}\right\}+\sum_{k} \mathcal{O}\left(\left|\partial_{x} \mathcal{W}\right|\right) \nu_{k}(U) .
\end{aligned}
$$

Combining 4.9), 4.10), and 4.11), we get

$$
\begin{aligned}
\partial_{t} \widetilde{\mathcal{W}} \equiv & L(U) \widetilde{\mathcal{W}}+\frac{e_{1}}{2} I-\frac{e_{2}}{8} I I \\
& +a(J(U)-J(V)) \partial_{x}^{4} \mathcal{V}+\sum_{k} \mathcal{O}\left(\left|\partial_{x}^{2} \mathcal{W}\right|+\left|\partial_{x} \mathcal{W}\right|\right) \nu_{k}(U)
\end{aligned}
$$

where

$$
\begin{aligned}
I & :=\partial_{x}^{2}\left\{J(U) \partial_{x}^{2}\left\{R\left(Z, U_{x}\right) U_{x}\right\}\right\}-R\left(J(U) \partial_{x}^{2} Z_{x x}, U_{x}\right) U_{x} \\
I I & :=\partial_{x}^{2}\left\{J(U) \partial_{x}^{2}\left\{R\left(J(U) U_{x}, U_{x}\right) J(U) Z\right\}\right\}+R\left(J(U) U_{x}, U_{x}\right) \partial_{x}^{2} Z_{x x}
\end{aligned}
$$

Remark 4.1. The structure of $I$ and $I I$ plays the essential role in our proof. Formally, we can write $\Lambda=\left(\Psi_{1}+\Psi_{2}\right) Z_{x x}$, where

$$
\Psi_{1}=-\frac{e_{1}}{2 a} R\left(\partial_{x}^{-2} \cdot U_{x}\right) U_{x}, \quad \Psi_{2}=\frac{e_{2}}{8 a} R\left(J(U) U_{x}, U_{x}\right) J(U) \partial_{x}^{-2} .
$$

Using the formulation, we can observe that

$$
-\frac{e_{1}}{2} I=\left[a \partial_{x}^{2}\left\{J(U) \partial_{x}^{2} \cdot\right\}, \Psi_{1}\right] Z_{x x}, \quad \frac{e_{2}}{8} I=\left[a \partial_{x}^{2}\left\{J(U) \partial_{x}^{2} \cdot\right\}, \Psi_{2}\right] Z_{x x},
$$

where the bracket $[\cdot, \cdot]$ denotes the commutator. 
We demonstrate the computation of $I$ and $I I$, where we repeatedly use the formula (2.24), (2.14). First, concerning $I$, a simple computation gives

$$
\begin{aligned}
& \partial_{x}\left\{R\left(Z, U_{x}\right) U_{x}\right\} \\
& =R\left(\partial_{x}\{P(U) Z\}, U_{x}\right) U_{x}+R\left(Z, U_{x x}\right) U_{x}+R\left(Z, U_{x}\right) U_{x x}-A\left(R\left(Z, U_{x}\right) U_{x}, U_{x}\right), \\
& \partial_{x}^{2}\left\{R\left(Z, U_{x}\right) U_{x}\right\} \\
& =R\left(\partial_{x}^{2}\{P(U) Z\}, U_{x}\right) U_{x}+2 R\left(\partial_{x}\{P(U) Z\}, U_{x x}\right) U_{x} \\
& \quad+2 R\left(\partial_{x}\{P(U) Z\}, U_{x}\right) U_{x x}-2 A(U)\left(R\left(\partial_{x}\{P(U) Z\}, U_{x}\right) U_{x}, U_{x}\right)+\mathcal{O}(|Z|) .
\end{aligned}
$$

Using $J(U) A(U)(\cdot, \cdot)=0$, we obtain

$$
\begin{aligned}
& J(U) \partial_{x}^{2}\left\{R\left(Z, U_{x}\right) U_{x}\right\} \\
& =J(U) R\left(\partial_{x}^{2}\{P(U) Z\}, U_{x}\right) U_{x}+2 J(U) R\left(\partial_{x}\{P(U) Z\}, U_{x x}\right) U_{x} \\
& \quad+2 J(U) R\left(\partial_{x}\{P(U) Z\}, U_{x}\right) U_{x x}+\mathcal{O}(|Z|) .
\end{aligned}
$$

From this and $J(U) A(U)(\cdot, \cdot)=0$, we continue the computation and obtain

$$
\begin{aligned}
\partial_{x}^{2}\{ & \left.J(U) \partial_{x}^{2}\left\{R\left(Z, U_{x}\right) U_{x}\right\}\right\} \\
= & J(U) R\left(\partial_{x}^{4}\{P(U) Z\}, U_{x}\right) U_{x}+2 \partial_{x}(J(U)) R\left(\partial_{x}^{3}\{P(U) Z\}, U_{x}\right) U_{x} \\
& +4 J(U) R\left(\partial_{x}^{3}\{P(U) Z\}, U_{x x}\right) U_{x}+4 J(U) R\left(\partial_{x}^{3}\{P(U) Z\}, U_{x}\right) U_{x x} \\
& +\mathcal{O}\left(|Z|+\left|Z_{x}\right|+\left|Z_{x x}\right|\right) .
\end{aligned}
$$

Here, we see

$$
\begin{aligned}
\partial_{x}^{3}\{P(U) Z\} & =P(U) \partial_{x} Z_{x x}+\mathcal{O}\left(|Z|+\left|Z_{x}\right|+\left|Z_{x x}\right|\right) \\
\partial_{x}^{4}\{P(U) Z\} & =P(U) \partial_{x}^{2} Z_{x x}+4 \partial_{x}(P(U)) \partial_{x} Z_{x x}+\mathcal{O}\left(|Z|+\left|Z_{x}\right|+\left|Z_{x x}\right|\right) \\
& =P(U) \partial_{x}^{2} Z_{x x}-4 A(U)\left(\partial_{x} Z_{x x}, U_{x}\right)+\mathcal{O}\left(|Z|+\left|Z_{x}\right|+\left|Z_{x x}\right|\right) .
\end{aligned}
$$

Thus we have $P(U) \partial_{x}^{3}\{P(U) Z\} \equiv P(U) \partial_{x} Z_{x x}$ and $P(U) \partial_{x}^{4}\{P(U) Z\} \equiv P(U) \partial_{x}^{2} Z_{x x}$. In addition, from (4.7) and $P(U) A(U)(\cdot, \cdot)=0$, we see $P(U) \partial_{x}^{3}\{P(U) Z\} \equiv P(U) \partial_{x} \mathcal{W}$ and $P(U) \partial_{x}^{4}\{P(U) Z\} \equiv P(U) \partial_{x}^{2} \mathcal{W}$. Substituting them into (4.15), we deduce

$$
\begin{aligned}
I \equiv & J(U) R\left(\partial_{x}^{2} \mathcal{W}, U_{x}\right) U_{x}-R\left(J(U) \partial_{x}^{2} \mathcal{W}, U_{x}\right) U_{x}+2 \partial_{x}(J(U)) R\left(\partial_{x} \mathcal{W}, U_{x}\right) U_{x} \\
& +4 J(U) R\left(\partial_{x} \mathcal{W}, U_{x x}\right) U_{x}+4 J(U) R\left(\partial_{x} \mathcal{W}, U_{x}\right) U_{x x} .
\end{aligned}
$$

The computation below is now standard. In the same way as above, using (2.18), (2.20), (2.21), 2.23), we deduce

$$
\begin{aligned}
J(U) R\left(\partial_{x}^{2} \mathcal{W}, U_{x}\right) U_{x}-R\left(J(U) \partial_{x}^{2} \mathcal{W}, U_{x}\right) U_{x} \\
=R\left(\partial_{x}^{2} \mathcal{W}, U_{x}\right) J(U) U_{x}+R\left(\partial_{x}^{2} \mathcal{W}, J(U) U_{x}\right) U_{x} \\
=-R\left(U_{x}, J(U) U_{x}\right) \partial_{x}^{2} \mathcal{W}-R\left(J(U) U_{x}, \partial_{x}^{2} \mathcal{W}\right) U_{x}+R\left(\partial_{x}^{2} \mathcal{W}, J(U) U_{x}\right) U_{x} \\
=2 R\left(\partial_{x}^{2} \mathcal{W}, J(U) U_{x}\right) U_{x}+R\left(J(U) U_{x}, U_{x}\right) \partial_{x}^{2} \mathcal{W} \\
\equiv 2 R\left(\partial_{x}^{2} \mathcal{W}, J(U) U_{x}\right) U_{x}+\partial_{x}\left\{R\left(J(U) U_{x}, U_{x}\right) \partial_{x} \mathcal{W}\right\} \\
\quad-2 R\left(J(U) \mathcal{U}, U_{x}\right) \partial_{x} \mathcal{W}+\sum_{k} \mathcal{O}\left(\left|\partial_{x} \mathcal{W}\right|\right) \nu_{k}(U)
\end{aligned}
$$

Using (2.8) and $R\left(\partial_{x} \mathcal{W}, U_{x}\right) U_{x} \perp \nu_{k}(U)$, we see

$$
2 \partial_{x}(J(U)) R\left(\partial_{x} \mathcal{W}, U_{x}\right) U_{x}=-2 A(U)\left(J(U) R\left(\partial_{x} \mathcal{W}, U_{x}\right) U_{x}\right.
$$




$$
=\sum_{k} \mathcal{O}\left(\left|\partial_{x} \mathcal{W}\right|\right) \nu_{k}(U)
$$

Using (2.26)-2.27), we see

$$
\begin{aligned}
& 4 J(U) R\left(\partial_{x} \mathcal{W}, U_{x x}\right) U_{x}+4 J(U) R\left(\partial_{x} \mathcal{W}, U_{x}\right) U_{x x} \\
& =4 R\left(\partial_{x} \mathcal{W}, \mathcal{U}\right) J(U) U_{x}+4 R\left(\partial_{x} \mathcal{W}, U_{x}\right) J(U) \mathcal{U} \\
& =4 R\left(J(U) \mathcal{U}, U_{x}\right) \partial_{x} \mathcal{W}+2\left(B_{2}(U)+B_{3}(U)\right) \partial_{x} \mathcal{W}
\end{aligned}
$$

Combining (4.16)-(4.18), we obtain

$$
\begin{aligned}
I \equiv & 2 R\left(\partial_{x}^{2} \mathcal{W}, J(U) U_{x}\right) U_{x}+\partial_{x}\left\{R\left(J(U) U_{x}, U_{x}\right) \partial_{x} \mathcal{W}\right\}+2 R\left(J(U) \mathcal{U}, U_{x}\right) \partial_{x} \mathcal{W} \\
& +2\left(B_{2}(U)+B_{3}(U)\right) \partial_{x} \mathcal{W}+\sum_{k} \mathcal{O}\left(\left|\partial_{x} \mathcal{W}\right|\right) \nu_{k}(U)
\end{aligned}
$$

Second, we compute $I I$. The way of the computation of $I I$ is now almost same as that for $I$. The result of the computation is as follows:

$$
\begin{aligned}
& \partial_{x}\left\{R\left(J(U) U_{x}, U_{x}\right) J(U) Z\right\}=\partial_{x}\left\{J(U) R\left(J(U) U_{x}, U_{x}\right) Z\right\} \\
& =J(U) R\left(J(U) U_{x}, U_{x}\right) \partial_{x}\{P(U) Z\}+\partial_{x}(J(U)) R\left(J(U) U_{x}, U_{x}\right) Z \\
& \quad+J(U) R\left(\partial_{x}\left\{J(U) U_{x}\right\}, U_{x}\right) Z+J(U) R\left(J(U) U_{x}, U_{x x}\right) Z \\
& \quad \partial_{x}^{2}\left\{R\left(J(U) U_{x}, U_{x}\right) J(U) Z\right\} \\
& =J(U) R\left(J(U) U_{x}, U_{x}\right) \partial_{x}^{2}\{P(U) Z\}+2 \partial_{x}(J(U)) R\left(J(U) U_{x}, U_{x}\right) \partial_{x}\{P(U) Z\} \\
& +2 J(U) R\left(\partial_{x}\left\{J(U) U_{x}\right\}, U_{x}\right) \partial_{x}\{P(U) Z\} \\
& +2 J(U) R\left(J(U) U_{x}, U_{x x}\right) \partial_{x}\{P(U) Z\}+\mathcal{O}(|Z|) . \\
& \quad J(U) \partial_{x}^{2}\left\{R\left(J(U) U_{x}, U_{x}\right) J(U) Z\right\} \\
& \quad=-R\left(J(U) U_{x}, U_{x}\right) \partial_{x}^{2}\{P(U) Z\}-2 R\left(\partial_{x}\left\{J(U) U_{x}\right\}, U_{x}\right) \partial_{x}\{P(U) Z\} \\
& \quad-2 R\left(J(U) U_{x}, U_{x x}\right) \partial_{x}\{P(U) Z\}+\mathcal{O}(|Z|) . \\
& \partial_{x}^{2}\left\{J(U) \partial_{x}^{2}\left\{R\left(J(U) U_{x}, U_{x}\right) J(U) Z\right\}\right\} \\
& =-R\left(J(U) U_{x}, U_{x}\right) \partial_{x}^{4}\{P(U) Z\}-4 R\left(\partial_{x}\left\{J(U) U_{x}\right\}, U_{x}\right) \partial_{x}^{3}\{P(U) Z\} \\
& -4 R\left(J(U) U_{x}, U_{x x}\right) \partial_{x}^{3}\{P(U) Z\} \\
& +\sum \mathcal{O}\left(\left|\partial_{x} \mathcal{W}\right|\right) \nu_{k}(U)+\mathcal{O}\left(|Z|+\left|Z_{x}\right|+\left|Z_{x x}\right|\right) \\
& \equiv-R\left(J(U) U_{x}, U_{x}\right) \partial_{x}^{2} \mathcal{W}-4 R\left(J(U) \mathcal{U}, U_{x}\right) \partial_{x} \mathcal{W} \\
& -4 R\left(J(U) U_{x}, \mathcal{U}\right) \partial_{x} \mathcal{W}+\sum_{k} \mathcal{O}\left(\left|\partial_{x} \mathcal{W}\right|\right) \nu_{k}(U) \\
& =-R\left(J(U) U_{x}, U_{x}\right) \partial_{x}^{2} \mathcal{W}-8 R\left(J(U) \mathcal{U}, U_{x}\right) \partial_{x} \mathcal{W}+\sum_{k} \mathcal{O}\left(\left|\partial_{x} \mathcal{W}\right|\right) \nu_{k}(U) .
\end{aligned}
$$

From (4.20) and $R\left(J(U) U_{x}, U_{x}\right) \partial_{x}^{2} Z_{x x} \equiv R\left(J(U) U_{x}, U_{x}\right) \partial_{x}^{2} \mathcal{W}$, we obtain

$$
I I \equiv-8 R\left(J(U) \mathcal{U}, U_{x}\right) \partial_{x} \mathcal{W}+\sum_{k} \mathcal{O}\left(\left|\partial_{x} \mathcal{W}\right|\right) \nu_{k}(U)
$$


Substituting (4.19) and (4.21) into 4.12), and using $\partial_{x}^{2} \widetilde{\mathcal{W}} \equiv \partial_{x}^{2} \mathcal{W}$ and $\partial_{x} \widetilde{\mathcal{W}} \equiv \partial_{x} \mathcal{W}$, we get

$$
\begin{aligned}
\partial_{t} \widetilde{\mathcal{W}} \equiv & L(U) \widetilde{\mathcal{W}}+e_{1} R\left(\partial_{x}^{2} \widetilde{\mathcal{W}}, J(U) U_{x}\right) U_{x}+\frac{e_{1}}{2} \partial_{x}\left\{R\left(J(U) U_{x}, U_{x}\right) \partial_{x} \widetilde{\mathcal{W}}\right\} \\
& +\left(e_{1}+e_{2}\right) R\left(J(U) \mathcal{U}, U_{x}\right) \partial_{x} \widetilde{\mathcal{W}}+e_{1}\left(B_{2}(U)+B_{3}(U)\right) \partial_{x} \widetilde{\mathcal{W}} \\
& +a(J(U)-J(V)) \partial_{x}^{4} \mathcal{V}+\sum_{k} \mathcal{O}\left(\left|\partial_{x}^{2} \widetilde{\mathcal{W}}\right|+\left|\partial_{x} \widetilde{\mathcal{W}}\right|\right) \nu_{k}(U)
\end{aligned}
$$

We now arrived at the estimate for $\left\langle\partial_{t} \widetilde{\mathcal{W}}, \widetilde{\mathcal{W}}\right\rangle$. Since $\widetilde{\mathcal{W}}=\mathcal{W}+\mathcal{O}(|Z|),\langle L(U) \widetilde{\mathcal{W}}, \widetilde{\mathcal{W}}\rangle$ has the same type of the estimate as that of $\langle L(U) \mathcal{W}, \mathcal{W}\rangle$ which is bounded by the right hand side of (3.64). More precisely, we deduce

$$
\begin{aligned}
\langle L(U) \widetilde{\mathcal{W}}, \widetilde{\mathcal{W}}\rangle \leqslant & (-a+b)\left\langle R\left(\partial_{x}^{2} \widetilde{\mathcal{W}}, J(U) U_{x}\right) U_{x}, \widetilde{\mathcal{W}}\right\rangle \\
& +\left(\frac{a}{2}-\frac{b}{2}+3 c\right)\left\langle R\left(J(U) \mathcal{U}, U_{x}\right) \partial_{x} \widetilde{\mathcal{W}}, \widetilde{\mathcal{W}}\right\rangle+C \widetilde{D}^{2}(t)
\end{aligned}
$$

for almost every $t$. The other terms (except the second and the fourth terms) of the right hand side of (4.22) can be handled in the same way as we obtain (3.52), (3.53), (3.56), and (3.51). Having them in mind, we take $e_{1}$ and $e_{2}$ so that

$$
e_{1}=a-b, \quad e_{2}=-e_{1}-\frac{a}{2}+\frac{b}{2}-3 c=-\frac{3 a}{2}+\frac{3 b}{2}-3 c .
$$

Then, we derive the following estimate

$$
\begin{aligned}
& \frac{1}{2} \frac{d}{d t}\|\widetilde{\mathcal{W}}\|_{L^{2}}^{2}=\left\langle\partial_{t} \widetilde{\mathcal{W}}, \widetilde{\mathcal{W}}\right\rangle \\
& \leqslant\left(e_{1}-a+b\right)\left\langle R\left(\partial_{x}^{2} \widetilde{\mathcal{W}}, J(U) U_{x}\right) U_{x}, \widetilde{\mathcal{W}}\right\rangle \\
& \quad+\left(e_{1}+e_{2}+\frac{a}{2}-\frac{b}{2}+3 c\right)\left\langle R\left(J(U) \mathcal{U}, U_{x}\right) \partial_{x} \widetilde{\mathcal{W}}, \widetilde{\mathcal{W}}\right\rangle+C \widetilde{D}^{2}(t) \\
& =C \widetilde{D}^{2}(t)
\end{aligned}
$$

for almost every $t \in(-T, T)$. This combined with (4.5) shows the desired estimate (4.4), and thus we complete the proof.

\section{ACKNOWLEDGMENTS}

The author has been supported by JSPS Grant-in-Aid for Scientific Research (C) Grant Number JP20K03703.

\section{REFERENCES}

[1] T. Akhunov, A sharp condition for the well-posedness of the linear KdV-type equation, Proc. Amer. Math. Soc. 142 (2014) 4207-4220.

[2] N.-H. Chang, J. Shatah, and K. Uhlenbeck, Schrödinger maps, Comm. Pure Appl. Math. 53 (2000) $590-602$.

[3] H. Chihara, Schrödinger flow into almost Hermitian manifolds, Bull. Lond. Math. Soc. 45 (2013) $37-51$.

[4] H. Chihara, Fourth-order dispersive systems on the one-dimensional torus, J. Pseudo-Differ. Oper. Appl. 6 (2015) 237-263.

[5] H. Chihara and E. Onodera, A third order dispersive flow for closed curves into almost Hermitian manifolds, J. Funct. Anal. 257 (2009) 388-404.

[6] H. Chihara and E. Onodera, A fourth-order dispersive flow into Kähler manifolds, Z. Anal. Anwend. 34 (2015) 221-249. 
[7] Q. Ding and Y. D. Wang, Vortex filament on symmetric Lie algebras and generalized bi-Schrödinger flows, Math. Z. 290 (2018) 167-193.

[8] W. Y. Ding and Y. D. Wang, Schrödinger flow of maps into symplectic manifolds, Sci. China Ser. A 41 (1998) 746-755.

[9] W. Y. Ding and Y. D. Wang, Local Schrödinger flow into Kähler manifolds, Sci. China Ser. A 44 (2001) $1446-1464$.

[10] J. Eells and J. H. Sampson, Harmonic mappings of Riemannian manifolds, Amer. J. Math. 86 (1964) 109?-160.

[11] Y. Fukumoto, Three-dimensional motion of a vortex filament and its relation to the localized induction hierarchy, Eur. Phys. J. B 29 (2002) 167-171.

[12] Y. Fukumoto and H. K. Moffatt, Motion and expansion of a viscous vortex ring. Part I. A higher-order asymptotic formula for the velocity, J. Fluid. Mech. 417 (2000) 1-45.

[13] B. Guo, M. Zeng, and F. Su, Periodic weak solutions for a classical one-dimensional isotropic biquadratic Heisenberg spin chain, J. Math. Anal. Appl. 330 (2007) 729-739.

[14] S. Herr, T. Lamm, T. Schmid, and R. Schnaubelt, Biharmonic wave maps: local wellposedness in high regularity, Nonlinearity 33 (2020) 2270-2305.

[15] C. C. Hsiung, Almost complex and complex structures, Series in Pure Mathematics, 20. World Scientific Publishing Co., Singapore, 1995. xvi+310 pp

[16] C. Kenig, T. Lamm, D. Pollack, G. Staffilani, and T. Toro, The Cauchy problem for Schrödinger flows, Discrete Contin. Dyn. Syst. 27 (2010) 389-439.

[17] N. Koiso, The vortex filament equation and a semilinear Schrödinger equation in a Hermitian symmetric space, Osaka J. Math. 34 (1997) 199-214.

[18] M. Lakshmanan, K. Porsezian, and M. Daniel, Effect of discreteness on the continuum limit of the Heisenberg spin chain, Phys. Lett. A. 133 (1988) 483-488.

[19] T. Lamm, Biharmonic Maps. PhD thesis, Albert-Ludwigs-Universität Freiburg im Breisgau, 2005.

[20] H. McGahagan, An approximation scheme for Schrödinger maps, Comm. Partial Differential Equations 32 (2007) 375-400.

[21] S. Mizohata, On the Cauchy Problem, Academic Press (1985)

[22] R. Mizuhara, The initial value problem for third and fourth order dispersive equations in one space dimension, Funkcial Ekvac 49 (2006) 1-38.

[23] A. Nahmod, J. Shatah, L. Vega, and C. Zeng, Schrödinger maps and their associated frame systems, Int. Math. Res. Not. IMRN 2007, no. 21, Art. ID rnm088, 29 pp.

[24] E. Onodera, Generalized Hasimoto transform of one-dimensional dispersive flows into compact Riemann surfaces, SIGMA Symmetry Integrability Geom. Methods Appl. 4 (2008) article No. 044, 10 pages.

[25] E. Onodera, A third-order dispersive flow for closed curves into Kähler manifolds, J. Geom. Anal. 18 (2008) 889-918.

[26] E. Onodera, A remark on the global existence of a third order dispersive flow into locally Hermitian symmetric spaces, Comm. Partial Differential Equations 35 (2010) 1130-1144.

[27] E. Onodera, The initial value problem for a fourth-order dispersive closed curve flow on the two-sphere, Proc. Roy. Soc. Edinburgh Sect. A. 147 (2017) 1243-1277.

[28] E. Onodera, A fourth-order dispersive flow equation for closed curves on compact Riemann surfaces, $J$. Geom. Anal. 27 (2017) 3339-3403.

[29] E. Onodera, Local existence of a fourth-order dispersive curve flow on locally Hermitian symmetric spaces and its application, Differential Geom. Appl. 67 (2019) 101560, 26pp.

[30] P. Y. H. Pang, H. .Y. Wang, and Y. D. Wang, Schrödinger flow on Hermitian locally symmetric spaces, Comm. Anal. Geom. 10 (2002) 653-681.

[31] J. C. Robinson, Infinite-Dimensional Dynamical Systems: An Introduction to Dissipative Parabolic PDEs and the Theory of Global Attractors, Cambridge Texts in Applied Mathematics, Cambridge University Press, Cambridge (2001)

[32] I. Rodnianski, Y. A. Rubinstein, and G. Staffilani, On the global well-posedness of the one-dimensional Schrödinger map flow, Analysis and PDE 2 (2009) 187-209.

[33] J. Segata, Refined energy inequality with application to well-posedness for the fourth order nonlinear Schrödinger type equation on torus. J. Differential Equations 252 (2012), 5994-6011. 
[34] J. Shatah and M. Struwe, Geometric wave equations, Courant Lecture Notes 2 (1998), Courant Institute, New York; second edition, AMS (2000)

[35] C. Song, The KdV curve and Schrödinger-Airy curve, Proc. Amer. Math. Soc. 140 (2012) 635-644.

[36] C. Song and Y. D. Wang, Uniqueness of Schrödinger flow on manifolds, Comm. Anal. Geom. 26 (2018) 217-235.

[37] P.L. Sulem, C. Sulem, and C.Bardos, On the continuous limit for a system of classical spins, Comm. Math. Phys. 107 (1986) 431-454.

[38] X. W. Sun and Y. D. Wang, Some geometric flows on Kähler manifolds, J. Partial Differ. Equ. 23 (2010) 203-208.

[39] X. W. Sun and Y. D. Wang, KdV geometric flows on Kähler manifolds, Internat. J. Math. 22 (2011) 1439-1500.

[40] X. W. Sun and Y. D. Wang, Geometric Schrödinger-Airy Flows on Kähler Manifolds, Acta Math. Sin. 29 (2013) 209-240.

[41] S. Tarama, $L^{2}$-well-posed Cauchy problem for fourth order dispersive equations on the line, Electron. J. Differential Equations 168 (2011) 1-11.

[42] R. Temam, Infinite-dimensional dynamical systems in mechanics and physics, second ed., Applied Mathematical Sciences, vol. 68, Springer-Verlag, New York (1997)

[43] C. L. Terng and K. Uhlenbeck, Schrödinger flow on Grassmannians, AMS/IP Stud. Adv. Math. 36 (2006) 235-256.

(Eiji Onodera) Department of Mathematics, Faculty of Science and Technology, Kochi UNIVERSITY, KOCHI 780-8520, JAPAN

E-mail address: onodera@kochi-u.ac.jp 